\title{
De Plantin à Deman Pour une histoire des pratiques d'édition en Belgique
}

Émergences, puis effondrements (ou replis vers des genres mineurs) : l'histoire des pratiques d'édition en Belgique bégaie. Des attitudes et des aptitudes toutefois se maintiennent, qui se transmettent peut-être de génération à génération. Telle est notre principale hypothèse, dans ce qui apparaîtra d'abord, nous l'espérons, comme une tentative, en en retraçant l'histoire à travers quatre de ses moments-phares, de contribuer à faire exister « l'édition en Belgique francophone ${ }^{1}$.

Encore faut-il s'entendre sur les termes. Non seulement l'activité éditoriale sur les territoires qui formeront la Belgique fluctue au gré des législations qui régentent le commerce de la librairie et de l'imprimerie dans les États ayant juridiction sur eux, mais parler d' « activité éditoriale » pour désigner la production de livres avant 1850 relève en grande partie de l'abus de langage. Le terme même d'édition ou d'éditeur est très peu utilisé avant le $\mathrm{XIX}^{\mathrm{e}}$ siècle en dehors de son sens strictement philologique. Certes, ce libraire d'ancien régime remplit bien des fonctions « éditoriales », en prenant des initiatives en matière de production, mais ces fonctions restent le plus souvent très limitées. Sauf exceptions, le libraire-éditeur agit au coup par coup, sans stratégie littéraire ou commerciale à moyen ou long terme. Lorsque l'éditeur au sens moderne apparaîtra - en gros, dans la première moitié du XIX ${ }^{\mathrm{e}}$ siècle, en synchronie significative avec la figure de l'Auteur - , il ne reprendra pas simplement les fonctions « éditoriales » dont s'occupait le libraire du $\mathrm{xvm}^{\mathrm{e}}$ : il les redéploiera, les systématisera, cependant que les métiers de la librairie et de l'imprimerie se définiront et se spécialiseront de leur côté. En simplifiant très fort, on peut proposer le schéma suivant, qu'il faudra garder à l'esprit dans tout ce qui suit : du Xv $v^{e}$ au $x^{e}$ siècles, le système de production du livre est successivement dominé par l'imprimerie (du xve au milieu du XviI $\left.{ }^{\mathrm{e}}\right)$, par la librairie (du milieu du XVII ${ }^{\mathrm{e}}$ au début du XIX ${ }^{\mathrm{e}}$ ) puis par l'édition (à partir des années 18301840). C'est vers l'imprimeur, puis le libraire, puis l'éditeur, que les auteurs et les manuscrits se dirigent successivement, c'est d'eux que viennent les offres faites aux lecteurs. C'est d'eux aussi, tour à tour, que proviennent les représentations légitimes du métier, telles qu'elles s'imposent à l'ensemble de la profession. Historiquement, il faut observer encore que l'instance qui acquiert la fonction-clé dans le système tend du même coup à se structurer, à se professionnaliser, mais aussi à engendrer et à entretenir une mythologie spécifique. Si les termes d'imprimeur ou de libraire resteront sémantiquement commutables jusqu'au début du $\mathrm{xx}^{\mathrm{e}}$ siècle, l'emploi préférentiel de l'un ou l'autre terme pour désigner la profession impliquera une représentation spécifique de celle-ci- pour l'un artisanale par exemple, pour l'autre davantage liée à la mise en contact du livre et du lecteur.

Une dernière précaution s'impose encore, avant d'entrer dans le vif du sujet. Vu les di-

1 Il est significatif, sous cet égard, que le seul ouvrage général portant sur l'histoire du livre en Belgique ait pris pour intitulé, non « Histoire de l'édition », mais Histoire du livre et de l'imprimerie en Belgique des origines à nos jours (Bruxelles, Le Musée du Livre, 6 vol., 1929-1934). S'articulent ici réflexe bibliophilique et représentation passéiste. 
mensions modestes auxquelles doit se résigner un article de revue, on ne s'étonnera pas que l'histoire que nous allons tenter de construire par bribes et morceaux s'arrête au seuil du $\mathrm{xx}^{\mathrm{e}}$ siècle ${ }^{2}$. On voudra bien ne pas s'étonner davantage du caractère parfois rapide et elliptique des portraits d'éditeurs brossés au passage, des contextes rétablis et des trajectoires profilées. À la recherche d'une logique de fonctionnement, dans les diverses formes qu'elle prend pour agir, nous nous sommes crus autorisés à faire l'économie des précisions à caractère historique qui abondent dans les monographies que nous avons mises à profit, et auxquelles nous renverrons le lecteur curieux. Notre hypothèse porte sur l'engendrement de comportements modèles et de catégories de représentation du métier d'éditeur, et sur leur transmission, moyennant transformations, à travers l'histoire.

\section{I.}

\section{Plantin ou la naissance de l'érudit-imprimeur}

L'histoire de la production de livres à l'époque moderne sur le territoire de la future Belgique s'ouvre sur un monument, dont l'ombre portée va s'étendre sur plusieurs siècles. Il s'agit bien sûr de Christophe Plantin, né vers 1520 et mort en $1589^{3}$. Toutefois, ce n'est pas tant sa trajectoire personnelle étonnante qui va nous intéresser au premier chef que ce que Plantin incarne : le passage de l'imprimerie réactive à l'imprimerie pro-active, c'est-à-dire à ce qu'on pourrait appeler la "proto-édition » (édition entendue au sens actuel du terme). Sans doute les "publishing printers", comme les appelle Léon Voet ${ }^{4}$, commencent-ils à apparaître ici et là en Europe. Ce sont le plus souvent des imprimeurs qui développent à la fois leurs fonctions commerciales (librairie et réseau de distribution) et leurs fonctions intellectuelles (en prenant des initiatives éditoriales et en entretenant des relations suivies avec les auteurs). Pour la France, on songe à Robert Estienne (Paris) ou à Étienne
Dolet (Lyon) ; en Italie, c'est le Vénitien Alde Manuce qui fait figure de grand précurseur. Mais Plantin offre l'exemple rare - unique en Belgique, période contemporaine comprise — d'un développement intégré des trois fonctions (imprimerie, librairie, édition), et à une échelle tout à fait extraordinaire pour son époque, tant en nombre de volumes imprimés, en importance des transactions commerciales qu'en densité du réseau intellectuel, social et politique installé en un temps très court. Qu'il ait été possible qu'un tel entrepreneur naisse et prospère à Anvers au cours de la seconde moitié du XVI ${ }^{\mathrm{e}}$ siècle en dit long d'une part sur le statut de cette ville à la Renaissance et d'autre part sur l'affaiblissement intellectuel et commercial des siècles ultérieurs, qui n'ont jamais permis la résurgence d'un tel prodige.

\section{1. « Labore et constantia»}

Soit donc un jeune relieur français de 28 ans arrivant à Anvers autour de 1548. Il a appris l'art de la reliure à Caen, avant d'émigrer vers la « ville monde " qu'est Anvers à cette époque. Sa maîtrise de la reliure d'art doit être très grande : très vite les commandes affluent et son nom commence à circuler parmi les patriciens de la ville. Cette renommée l'aide sans doute à obtenir rapidement son privilège d'imprimeur. Dès 1555 , le relieur imprime : des ouvrages savants, des manuels d'éducation pour jeunes filles (son premier livre : L'Institution d' une fille de noble maison, par Giovanni Michele Bonto), mais aussi, en grand secret, les ouvrages hérétiques d'une secte anabaptiste fondée par un certain Hendrik Niclaes.

Sincèrement catholique, mais d'une foi mysticisante proche du calvinisme piétiste, Plantin ressemble beaucoup à ces entrepreneurs protestants dont Max Weber a construit l'idéal-type. Sa devise même, «Labore et Constantia », représentée sous la forme d'un compas en mouvement, peut être vue comme une expression de cet « ascétisme séculier » qui est au cœur du

2 Cf. DuRAnd (P.) et Winkin (Y.), Marché éditorial et démarches d'écrivains. Un état des lieux et des forces de l'édition littéraire en Communauté française de Belgique. Bruxelles, Direction générale de la Culture, 1996, 307 p.

3 L'ouvrage de référence sur Plantin et ses successeurs est celui de Léon VoET :The Golden Compasses. A History and Evaluation of the Printing and Publishing Activities of the Officina Plantiniana at Antwerp. Amsterdam, Vangendt, 1969, 2 vol.

4 Voet (L.), op.cit., vol. 2, p. 283. Voir aussi sur ce point FebVre (L.) et Martin (H.-J.), L'Apparition du livre. Paris, Albin Michel, 1958, pp. 244-259. 
capitalisme moderne ${ }^{5}$. Travailleur acharné, Plantin a dû certainement l'être, vu ses chiffres de production : en 1555,10 titres nouveaux imprimés ; en 1556, 12 ; en 1557, 21 ; en 1558, 23 ; en 1559,13 ; en 1560,13 ; en 1561,28 ; en 1562,21 et, de 1563 à 1567,155 . Près de trois cents titres en treize ans : la cadence est impressionnante quand on sait que l'un de ses principaux concurrents anversois, Willem Silvius, n'en a produit que cent vingt en 21 ans de carrière (de 1559 à 1580). Dès 1561, Plantin fait tourner quatre presses. Puissance de production exceptionnelle elle aussi : $\mathrm{au} \mathrm{xvII}^{\mathrm{e}}$ siècle à $\mathrm{Pa}$ ris, cinq imprimeurs à peine dépasseront ce nombre. Plantin est donc bien, en effet, un «météore dans le ciel d'Anvers » (L. Voet). À la fois imprimeur, librairie, éditeur, mais encore marchand de gravures, de cartes, de globes, de papier, de cuir, de dentelle, il continue par ailleurs de fournir à la commande des reliures très raffinées, des miroirs et des cassettes emblasonnées. Son étonnante ascension commerciale (et sociale), il la doit à ses capacités de travail, mais aussi à son habileté à se mettre au service du pouvoir, tout en se ménageant des portes de sortie. En 1562-63, Marguerite de Parme, gouverneur général de Philippe II aux Pays-Bas, ordonne une enquête sur les origines d'un catéchisme calviniste, Briefve instruction pour prier. Plantin est soupçonné. Il se réfugie à Paris, attendant que l'orage passe. L'enquête conclut que trois de ses ouvriers ont imprimé l'ouvrage à son insu. Quatre ans plus tard, un scénario similaire se répétera.

De retour à Anvers, Plantin s'associe à quatre « investisseurs », qui lui garantissent un salaire annuel ${ }^{6}$. Les capitaux investis vont permettre à l'imprimerie d'utiliser dès 1566 sept presses autour desquelles s'activent trente-trois ouvriers. Les productions sont non seulement religieuses et littéraires (auteurs classiques) mais encore scientifiques : traités de médecine (Vésale), études de botanique, etc. Subitement, tout s'arrête. Plantin s'aperçoit que ses associés sont des hérétiques. En fait, il participe avec eux à la constitution d'une imprimerie à Vianen, le quar- tier général des Iconoclastes. Puis, il prend peur quand le duc d'Albe arrive à Bruxelles en août 1567. Il proclame alors avec véhémence qu'il a été trompé par ses associés et qu'il est en fait un catholique fidèle, tout dévoué à la Couronne espagnole (tandis qu'en secret, il continue à correspondre avec eux). Il courtise ainsi de façon systématique deux dignitaires du régime : le Cardinal Granvelle, archevêque de Malines, et Gabriel de Cayas, secrétaire de Philippe II, auquel il propose la publication d'une « Bible polyglotte ». Le projet en est finalement accepté par Philippe II, qui lui octroie en 1568 un crédit de 12.000 florins et lui envoie son chapelain, le moine bénédictin Arias Montanus, théologien déjà célèbre pour ses interventions au Concile de Trente, pour diriger l'entreprise, qui va vite se révéler gigantesque : cinq langues (latin, grec, hébreu, chaldéen, syriaque), huit volumes infolio, dont cinq pour les textes et trois, très illustrés, pour les commentaires et notes.

La période de préparation et d'impression de la Biblia Polyglotta (1568-1572) marquera le début de l'expansion de l'Officina Plantiniana, qui devient peu à peu une véritable manufacture de livres. Utilisant ses entrées auprès du Pape (Granvelle) et auprès de Philippe II (Montanus), Plantin parvient à obtenir plusieurs monopoles d'impression des nouveaux livres religieux conformes aux décisions du Concile de Trente. Les privilèges papaux (qui ne valent que pour les Pays-Bas et que des imprimeurs de Liège et de Cologne ne respectent pas) sont moins bénéfiques que ceux conférés par le Roi d'Espagne. En 1571, celui-ci accorde à Plantin le monopole d'impression et de vente des bréviaires et missels destinés à l'Espagne et à ses territoires. Le marché est fabuleux et Plantin y consacre tous ses efforts, en augmentant le nombre de presses en service ( 46 ouvriers s'occupent de 13 presses en 1572) et en diminuant la production d'autres livres (qui restent néanmoins variés dans leur production : auteurs classiques, traités de droit et de théologie, travaux de Juste Lipse, etc.). Son hégémonie est encore renforcée par l'octroi du titre de prototypogra-

5 «L'évaluation religieuse du travail sans relâche, continu, systématique, dans une profession séculière, comme moyen ascétique le plus élevé et à la fois preuve la plus sûre, la plus évidente de régénération et de foi authentique, a pu constituer le plus puissant levier qui se puisse imaginer dans l'expression de cette conception de la vie que nous avons appelée, ici, l'esprit du capitalisme » (WEBER (M.), L'Éthique protestante et l'esprit du capitalisme (1920). Paris, Plon, 1964, p. 236).

6 L'association entre Plantin et ces quatre bailleurs de fonds paraît très moderne : elle évoque dans son principe la «S.A. » avec « administrateur délégué ». 
phe de Philippe II ${ }^{7}$. Hiérarchiquement à la tête de tous les imprimeurs des Pays-Bas, Plantin n'aura pas grand-chose à faire pour tenir son rang d'architypographe, mais ce titre de gloire ornera longtemps ses frontispices.

L'année 1572, particulièrement turbulente sur le plan politique (sac de Malines par les Espagnols et révolte rampante contre le régime espagnol à travers le pays), révèle à nouveau le caractère louvoyant du bonhomme. Sommé par de Cayas d'expliquer pourquoi les missels commandés par le Roi n'arrivent plus, Plantin répond que ses ouvriers se sont mis en grève et qu'il a répliqué par un lock out. En fait, il a licencié 33 de ses 46 ouvriers parce que la situation politique l'inquiète et qu'il songe à fermer son entreprise pour s'installer à Paris. On retrouve encore la dérobade typique de Plantin, alors que la principale responsabilité lui revient. Une fortune meilleure refait surface dès 1573 . Philippe II paie plus régulièrement et passe d'autres commandes. Plantin, pour sa part, cherche à moins dépendre de ce seul commanditaire et redéveloppe l'édition d'ouvrages divers. Comme le note Voet, il calcule très bien ses coups (et ses coûts) ${ }^{8}$. Prudence payante : en 1574 , Plantin possède seize presses $(55$ ouvriers travaillent à l'imprimerie, sur un total de 150 emplois), devenant ainsi « le plus grand imprimeur de la période s'étendant de la Renaissance à la fin du XvIII ${ }^{\mathrm{e}}$ siècle ${ }^{9}$.

Et puis la Furie espagnole se déchaîne à Anvers le 4 novembre 1576. Plantin est rançonné à plusieurs reprises mais échappe aux massacres. Anvers passe aux mains des troupes protestantes des États Généraux en 1577. L'imprimeur reprend peu à peu ses activités, en empruntant de l'argent à divers amis et en modifiant une fois de plus ses allégeances. Les énormes commandes de Philippe II se sont taries, il en faut d'autres. Ce seront d'une part des ouvrages à la commande, « à compte d'auteur » ou pour le compte d'autres éditeurs, d'autre part des travaux d'impression pour les États Généraux, dont il devient à sa demande imprimeur officiel en 1578, pour la Ville d'Anvers, dont il devient également imprimeur officiel (en 1579), et pour le Duc d'Anjou, à qui il sollicite l'hon- neur d'être son imprimeur lorsqu'il entre dans la ville en 1582 . Tout en continuant de correspondre discrètement avec ses anciens protecteurs espagnols, à qui il explique qu'il est obligé de composer avec le nouveau pouvoir afin de sauver son entreprise mais que son cœur reste fidèle à la foi catholique et au Roi d'Espagne, il accueille Guillaume d'Orange en 1579 par des vers enthousiastes et publie en 1582 La joyeuse et magnifique entrée de Monseigneur François en l'honneur du Duc d'Anjou. Il publie ainsi nombre d'études calvinistes et d'écrits anti-espagnols, dont le célèbre ouvrage de Bartolomeus de las Casas, Tyrannies et cruautéz des Espagnols, perpetrées és Indes Occidentales (1579). Il prend certes quelque précaution pour rester neutre (notamment en publiant sous le nom de son beau-fils, Frans Raphelengius, très proche des Calvinistes, ou en publiant en grand secret, par exemple dans le cas des travaux hérétiques de Barrefelt, un dissident de la secte de $\mathrm{H}$. Niclaes). Mais son image se trouble en des temps qui sont troubles eux aussi. En 1583, Plantin quitte Anvers pour l'Université de Leyde où enseigne Juste Lipse. Il a confié la direction des affaires anversoises à ses deux gendres, Jan Moretus et Frans Raphelengius. Sans doute a-til l'intention de revenir à Anvers, mais lorsque l'atmosphère y sera moins lourde. En attendant, il accepte le poste d'imprimeur officiel de l'Université et commence à produire divers livres.

En 1585, nouveau revirement : Plantin rentre à Anvers, que les troupes d'Alexandre Farnèse viennent de reconquérir. Il est à nouveau au service des Espagnols, qui le confirment dans ses fonctions officielles antérieures. Ses dernières années, cependant, seront difficiles. Il ne travaillera plus qu'avec une demi-douzaine de presses et quelques hommes. Mais il produira encore quelques très beaux ouvrages, telle l'édition espagnole du Theatrum Orbis Terrarum d'Ortelius, avant de mourir le $1^{\text {er }}$ juillet 1589 . En 34 ans d'activité, il aura publié 1.500 ouvrages.

\section{Un « banquier symbolique»?}

Pour Henri Pirenne, Plantin est un « capita-

7 « Avons ordonné et ordonnons... sera créé et institué un prototypographe, ou premier typographe, pour avoir superintendence sur le fait d'icelle imprimerye. Qui aura auctorité d'examiner et approuver les maistres et ouvriers de l'Imprimerye de nos-dictz pays de pardeça » (Ordonnance du 19 mai 1570).

9 VOET (L.), op.cit., vol. 1, p. 83. 
liste de génie ${ }^{10}$. Pour d'autres, un opportuniste faisant flèche de tout bois. Pour Voet, « un homme d'affaires rempli de soucis financiers qui, pris dans la tourmente d'un furieux conflit politique et religieux, a essayé de tirer le meilleur parti d'une mauvaise situation $"{ }^{11}$. Pour nous, Plantin est un étonnant "érudit-imprimeur » (selon l'expression de E.-L. Eisenstein ${ }^{12}$ ), qui préfigure l'éditeur moderne par nombre d'éléments de sa trajectoire. Et sa capacité même à s'adapter aux circonstances, à suivre les « modes », oserait-on dire, n'est pas la moindre de ses attitudes modernes.

Contrairement à la plupart des imprimeurs de l'époque, qui reproduisent simplement les manuscrits que les auteurs leur apportent, Plantin fera preuve d'initiative à plusieurs reprises, soit en recherchant des manuscrits égarés ${ }^{13}$, soit en élaborant d'abord le projet de l'ouvrage et en cherchant ensuite l'auteur qui pourrait le réaliser. Le cas le plus patent est celui de la Bible polyglotte, dont il propose l'idée à Philippe II en 1568. Mais il en est d'autres, moins connus quoique plus significatifs encore, parce que Plantin paie lui-même les auteurs (ou plus exactement les «éditeurs ", au sens de "préparateurs de textes »). C'est ainsi que Joannes Isaac Levita de Cologne, qui édite le Thesaurus linguae sanctae de Pagnini, est nourri et logé par Plantin pendant près d'un an (1563-64) et reçoit en outre environ 70 florins en livres et en liquide ${ }^{14}$. Certes, il ne s'agit pas de commandes intégrales. Le manuscrit existe le plus souvent sous une forme ou sous une autre et le travail consiste à « le mettre en ordre pour l'imprimer » (lettre de Plantin à Granvelle, décem- bre 1567). Mais la démarche éditoriale, au sens moderne, est nette. En outre, Plantin utilise son réseau d'amis pour se faire conseiller dans le choix et la préparation de certains manuscrits. Dans cette même lettre à Granvelle, on peut lire : « Vrai est que, passé 3 ans, j'avais prié plusieurs personnages doctes de me donner leurs observations sur ledit auteur et avoir obtenu quelques corrections $» 15$.

Du point de vue qui nous occupe, les rapports que Plantin entretient avec ses auteurs sont également fort intéressants. Tout d'abord, il semble qu'il reçoive nombre de manuscrits et de propositions et qu'il en refuse au moins la moitié, non tant pour des raisons d'intérêt que pour des raisons de coût. Si l'auteur est prêt à tout payer, Plantin imprime sans discuter : on est encore loin ici de l'éditeur moderne qui refuse de travailler à compte d'auteur. En fait, cette dernière pratique est de règle $a u x^{2}{ }^{e}$ siècle : l'auteur contribue à l'impression de son livre en achetant à l'avance un certain nombre d'exemplaires au prix de revient. Même Montanus devra ainsi acheter 50 exemplaires (pour $200 \mathrm{fl}$.) de ses Elucidationes in omnia Sanctorum Apostolorum scripta ${ }^{16}$. Plantin veut ainsi s'assurer de rentrer dans ses frais, même si l'ouvrage est contrefait avant que l'édition plantinienne en soit écoulée ${ }^{17}$. Il ne prendra de risques financiers qu'en de rares circonstances : en cas de succès assuré (e.g. Ortelius) ou de patronage politique (e.g. auteurs recommandés par Granvelle). Plantin semble donc avoir généralement travaillé au coup par coup. Chaque livre est conçu de manière indépendante : les notions de collection, de série, sinon d'œuvre

10 Pirenne parle d'Anvers comme d'une ville qui sait, dès la première moitié du $\mathrm{xvI}^{\mathrm{e}}$ siècle, combiner développement capitaliste et développement technique pour devenir une grande cité manufacturière : « L'esprit capitaliste qui y règne et y rend impossible le conservatisme routinier que le régime corporatif impose à d'autres villes, lui permet de profiter des perfectionnements apportés à la technique industrielle " (Histoire de Belgique. Bruxelles, Lamertin, 1912, p. 276).

11 Voet (L.), op.cit., vol. 1, p. 124.

12 EISENSTEIN (E.-L.), « Le livre et la culture savante », dans Histoire de l' édition française, op.cit., I, pp. 563583.

13 Voet rapporte qu'il a fait rechercher des manuscrits à Vienne et en Espagne, qu'il a négocié des prêts de manuscrits avec des abbayes, etc. (op.cit., vol. 1, pp. 383-384).

14 Exemple donné par VOET (L.), op.cit., vol. II, p. 286, note 4.

15 Cité par VOET (L.), op.cit., vol. II, p. 286, note 4 (p. 287).

16 Exemple donné par Voet (L.), op.cit., vol. II, p. 295.

17 Sa correspondance le montre souvent en train d'essayer de faire respecter ses privilèges, qui ne valent le plus souvent que pour les Pays-Bas espagnols : « Combien de longs temps j'aye asses entendu les menasses de vos aliés de faire contrefaire à Liége les sortes dont j'ay ou j'aurray privilège par deça, et que je sceusse cela estre de longtemps projetté et commencé... " (Lettre au marchand-imprimeur liégeois Hendrik van den Hove, début avril 1571, citée par Voet (L.), op.cit., vol. II, p. 291, note 1.) 
d'un auteur suivi, ne sont pas encore des catégories opérationnelles. Ce qui ne veut pas dire que la pensée prospective n'existe pas : l'édition de la Bible polyglotte s'étale sur plusieurs années, les stocks eux-mêmes sont autant d'engagements sur l'avenir (alors que l'époque, particulièrement troublée, est peu propice aux plans d'avenir), l'idée d'une souscription ne lui est pas inconnue. Ainsi, Plantin se situe à mi-chemin entre l'imprimerie classique, qui agit au coup par coup, et l'édition moderne, qui raisonne à long terme. En outre, contrairement à l'éditeur littéraire moderne qui, surtout à partir du $\mathrm{XIX}^{\mathrm{e}}$ siècle, va euphémiser son rapport à l'argent en se plaçant du côté des artistes, de la « pensée » et de la «culture », Plantin reste en toutes circonstances un "shrewd dealer" (comme dit Voet) : un florin est un florin. Ce rapport franc et maîtrisé à l'argent, qui se voit autant dans sa comptabilité, extraordinairement minutieuse, que dans sa correspondance, où l'on ne cesse de parler chiffres, peut être mis en rapport avec son éthos fondamentalement puritain (même si sa religion professée est catholique), de même que sa foi en l'avenir et sa volonté de maintenir son entreprise à flot envers et contre tout. Nombreux sont les commentateurs qui ont vu en Plantin à la fois un homme d'affaires et un ascète « à tête de huguenot ». On a plus rarement fait le rapprochement entre ces deux traits pour voir en Plantin une illustration de la thèse de Weber sur la naissance du capitalisme moderne. Ce que nous faisons ici, mais en soulignant un aspect du personnage qui n'appartient pas à l'idéal-type de l'entrepreneur wébérien : c'est un « intellectuel », dont la maison ressemble apparemment à un colloque international permanent ${ }^{18}$. Si les savants de passage ne sont pas des Pays-Bas, ils sont de France, d'Espagne, d'Italie, d'Allemagne, d'Europe centrale. Et quand ils ne vont pas chez Plantin, Plantin va chez eux : de Paris à Francfort, de Liège (Torrentius) à Duisburg (Mercator). Plantin correspond donc bien à l'" érudit-imprimeur » du XVI ${ }^{\mathrm{e}}$ siècle dont parle E. Eisenstein ${ }^{19}$ : son officine fait fonction de « maison internationale miniature ${ }^{20}$, contribuant du coup à la formation d'un nouvel éthos « cosmopolite, œcuménique et tolérant ${ }^{21}$.

Reste une question, que les savants travaux de Voet et de Eisenstein n'abordent pas : la dimension intellectuelle de l'Officina Plantiniana suffit-elle à faire de Plantin un « banquier symbolique » 22 ? Un ouvrage frappé du Compas d'Or donne-t-il à son auteur une légitimité plus grande que s'il sort des presses de Silvius, Foppens et autres imprimeurs contemporains? $\mathrm{Vu}$ le rayonnement international de Plantin, on pourrait le croire. Mais il semble qu'une dissociation reste présente entre l'imprimeur Plantin et l'humaniste Plantin, comme si ses fonctions marchandes oblitéraient ses fonctions éditoriales. Comme si, surtout, les temps n'étaient pas mûrs encore pour l'émergence de telles fonctions. Schücking suggère ainsi que l'éditeur comme agent de légitimation de l'œuvre n'émerge en Angleterre et en Allemagne qu'au $\mathrm{XVIII}^{\mathrm{e}}$ siècle $^{23}$. Demander dès lors à Plantin de « griffer » les travaux qu'il produit est peut-être aller trop loin dans la démonstration de sa mo-

18 Voet consacre un long chapitre de sa monumentale étude à « la maison Plantin comme centre humaniste » : « There is harldy a single scholar of any distinction from the Netherlands who does not figure in these lettres in some degree of relationship with the Plantin house. Some appear occasionally or casually, others with greater frequency : Louvain professors, bishops and abbots, theologians and humanists in clerical garb, doctors in medicine and law; scholars who had remained in the Low Countries, scholars who wondered through Europe or had found their spheres of activity at the Imperial courts of Vienna or Prague, in Spain or Italy » (op.cit., vol. I, p. 370).

19 «C'est ainsi que naquit un "homme nouveau”, l'érudit-imprimeur, capable de manier les machines et de vendre ses produits alors qu'il mettait des textes au point, créait des sociétés savantes, lançait des artistes et des écrivains, faisait progresser la collecte des données et les diverses disciplines savantes » (EISENSTEIN (E.L.), « Le livre et la culture savante », art.cit., p. 364).

20 C'est l'expression même de E.L. Eisenstein dans The Printing Press as an Agent of Change, I. Cambridge University Press, 1979, p. 139.

21 Ibid, p. 140 et p. 443.

22 Bourdieu (P.), « La production de la croyance », Actes de la recherche en sciences sociales, $\mathrm{n}^{\circ} 13$, fév. 1977 , pp. 3-44.

23 Il cite à ce propos ces vers de Richard Graves : « In vaint the poets from their mine/ Extract the shining mass,/Till Dodsley's Mint has stamped the coin/And hids the sterling pass. » - ScHücKING (L.), A Sociology of Literary Taste. London, Routledge and Kegan Paul, 1948, p. 51. 
dernité éditoriale. Mais on ne peut pas non plus, en sens inverse, en évacuer totalement l'idée, ainsi qu'en témoigne une remarque incidente de Montaigne au troisième livre de ses Essais ${ }^{24}$.

Quoi qu'il en soit, les successeurs de Plantin vont peu à peu ternir l'aura intellectuelle du fondateur pour n'être plus que des marchandsimprimeurs, de plus en plus puissants sur la scène sociale mais de plus en plus marginalisés sur la scène intellectuelle. Jean Moretus reprendra les succès du beau-père : Juste Lipse, les ouvrages de botanique, les ouvrages religieux (dont les énormes Annales ecclesiastici de Baronius). Ce sont des ouvrages très bien illustrés (notamment par Rubens, ami du petit-fils de Plantin). Ce sont aussi des ouvrages remarquablement bien diffusés, grâce à un système d'échanges entre grands libraires européens, de Londres à Vienne et Cracovie, de Copenhague à Paris et Madrid (afin d'éviter le transfert de numéraire, toujours aléatoire, ces libraires internationaux, dont font partie les Moretus, travaillent beaucoup par trocs et lettres de change). Mais ce sont des livres sans génie, dont la dimension créatrice reste faible, par rapport à l'inventivité intellectuelle et graphique de Plantin lui-même. Tout se passe comme si se mettait en place, dès le début, du XVII ${ }^{\mathrm{e}}$, la structure éditoriale de la future Belgique : elle importe et exporte beaucoup, elle imprime bien et puissamment, mais elle ne crée pas grand-chose. En cela, la production anversoise $\operatorname{des}_{\mathrm{XVI}}^{\mathrm{e}}$ et $\mathrm{XVII}^{\mathrm{e}}$ reste proche de cette littérature provinciale qu'on a appelée "les livres d'usage ». Ce sont des ouvrages répondant aux besoins quotidiens - matériels, intellectuels, spirituels — des ecclésiastiques, des gens de justice, des enseignants et de leurs élèves. Ce sont des livres précis, rigoureux, sévèrement contrôlés. Mais les livres d'usage anversois, contrairement à leurs homologues des provinces françaises, dont la diffusion est très locale, seront envoyés aux quatre coins de l'Europe, et au delà puisque le soleil ne se couche jamais sur l'empire espagnol. Pour le dire en une formule brutale, la banalité du livre « belge » sera le prix de son universalité.

\section{II.}

\section{Bassompierre ou la franche contrefaçon}

L'anecdote se passe en 1767 à Liège ${ }^{25}$. JeanFrançois Marmontel revient de Spa et s'arrête en la Cité Ardente :

À Liège, où nous avions couché, je vis entrer chez moi, le matin, un bourgeois d'assez bonne mine, et qui me dit : “ Monsieur, j'ai appris hier au soir que vous étiez ici ; je vous ai de grandes obligations, je viens vous en remercier. Mon nom est Bassompierre ; je suis imprimeur-libraire dans cette ville ; j'imprime vos ouvrages dont j'ai un grand débit dans toute l'Allemagne. J'ai déjà fait quatre éditions copieuses de vos Contes moraux; je suis à la troisième édition de Bélisaire. Quoi! Monsieur, lui dis-je, en l'interrompant, vous me volez le fruit de mon travail et vous venez vous en vanter à moi ! — Bon! reprit-il, vos privilèges ne s'étendent point jusqu'ici ; Liège est un pays de

24 À lire cette page de Montaigne - et même s'il évoque les cas Plantin et Vascosan pour regretter l'affiche de respectabilité intellectuelle dont ces imprimeurs sont susceptibles de recouvrir des ouvrages parfois quelconques, - il semble bien en effet que Plantin a pu apparaitre auprès des lettrés du temps comme une sorte de banquier symbolique : «Que ferons nous à ce peuple qui ne fait recepte que de tesmoignages imprimez, qui ne croit les hommes s'ils ne sont en livre, ny la vérité si elle n'est d'aage competant? Nous mettons en dignité nos bestises quand nous les mettons en moule. Il y a bien pour luy autre poix de dire : je l'ai leu, que si vous dictes : je l'ay ouy dire. Mais moy, qui ne mescrois non plus la bouche que la main des hommes, et qui scay qu'on escript autant indiscretement qu'on parle, et qui estime ce siecle comme un autre passé, j'allegue aussi volontiers un mien amy que Aulugele et que Macrobe, et ce que j'ay veu que ce qu'ils ont escrit. Et, comme ils tiennent de la vertu qu'elle n'est pas plus grande pour estre plus longue, j'estime de même la verité que, pour estre plus vieille, elle n'est pas plus sage. Je dis souvent que c'est pure sottise qui nous fait courir apres les exemples estranges et scholastiques. Leur fertilité est pareille à cette heure à celle du temps d'Homere et de Platon. Mais n'est-ce pas que nous cherchons plus l'honneur de l'allegation que la verité du discours ? comme si c'estoit plus d'emprunter de la boutique de Vascosan ou de Plantin nos preuves, que de ce qui se voit en nostre village » (Les Essais [édition Villey], Livre III, chap. XIII. Paris, P.U.F., 1988, p. 1081).

25 On la trouve dans Francotte (H.), La Propagande des Encyclopédistes français au Pays de Liège (17501?90). Bruxelles, Hayez, 1880, pp. 90-100. Joseph Brassine la reprend dans « L'imprimerie à Liège jusqu'à la fin de l'Ancien Régime ", dans Histoire du livre et de l' imprimerie ..., op.cit. 
franchise. Nous avons droit d'imprimer tout ce qu'il y a de bon ; c'est là notre commerce. Qu'on ne vous vole point en France, où vous êtes privilégié, vous serez encore assez riche. Faites-moi donc la grâce de venir déjeuner chez moi ; vous verrez une des belles imprimeries de l'Europe, et vous serez content de la manière dont vos ouvrages y sont exécutés ${ }^{26}$.

Liège est effectivement un pays de franchise où l'imprimerie se développe $\mathrm{au} \mathrm{XVIII}^{\mathrm{e}}$ siècle grâce à une' pratique tout aussi franche de la contrefaçon. Jean-François Bassompierre n'a rien d'un contrebandier. Il a des titres (il est « Imprimeur du Conseil Privé » du Prince-Évêque), il a pignon sur rue (il possède une belle librairie rue Neuvice, « À l'Arbre d'Or »), il fait des affaires importantes (de son imprimerie partent des livres qui se vendent autant à Paris qu'à Leipzig et Francfort). Et parmi ses livres, il y a bien sûr de nombreux « piratages » d'œuvres étrangères. Rien d'illicite là-dedans, Bassompierre ne s'en cache pas, pas plus que ses confrères : la contrefaçon est ouverte, joyeuse même. Mais parce que son histoire a toujours été écrite d'un point de vue français (ou par des bibliophiles belges drapés dans leurs vertus morales), la contrefaçon est perçue, aujourd'hui encore, comme une des pages sombres de l'histoire de la production du livre dans les « territoires belgiques » ${ }^{27}$. Les développements qui suivent se donnent donc pour but, non de réhabiliter la contrefaçon, mais de l'envisager comme une des « lignes de faîte " de l'édition en Belgique.

Les privilèges ont deux défauts : d'une part ils ne valent que pour un temps limité et qu'au sein du territoire couvert par l'autorité qui les garantit ; d'autre part ils offrent à cette autorité la possibilité d'un contrôle permanent sur la production de livres : le revers du privilège c'est la censure, dans laquelle baignera tout l'Ancien Régime. On comprend aisément que la contrefaçon se développe dans les entités qui échappent aux pouvoirs forts, notamment dans les provinces françaises et belges, dans les princi- pautés libres (Avignon, Bouillon, Trévoux, Liège) et dans les villes suisses (Genève, Neufchâtel, Lausanne). La contrefaçon participe donc de mouvements plus vastes, qui s'étendent à toute l'Europe. C'est dans ce contexte qu'il faut en saisir la portée pour la future édition belge. Il faut se garder de croire, cependant, que le développement des imprimeries et libraires belges serait uniquement dû à la contrefaçon d'ouvrages français ou hollandais. Les Moretus, les Verdussen, les Meursius, les Nutius, qui dominent les $\mathrm{XVII}^{\mathrm{e}}$ et $\mathrm{XVIII}{ }^{\mathrm{e}}$ siècles anversois, ne semblent guère recourir à cette pratique. La raison en est qu'ils comptent parmi les grands bourgeois du commerce européen du livre, proches des pouvoirs civils et ecclésiastiques, qui les ont richement dotés en privilèges. Ils sont par là les homologues de ces dynasties parisiennes d'imprimeurs-libraires que Louis XIV et Colbert ont protégées. De fait, les productions anversoises vont souvent faire l'objet de contrefaçons, notamment de la part d'imprimeurs lyonnais et hollandais de livres religieux. À Bruxelles par contre, même les plus établis des imprimeurslibraires, tels les Foppens et les Fricx, pratiquent au XVII ${ }^{\mathrm{e}}$ la contrefaçon sur une grande échelle. Ainsi, « dès que paraissait une édition intéressante en Hollande ou à Paris, on la reproduisait à Bruxelles » ${ }^{28}$. Ces contrefaçons sont loin d'être typographiquement médiocres, surtout quand elles sortent des ateliers Foppens, qui se spécialisent dans la reproduction de livres en format in-12, à l'instar de ceux produits par la maison Elzevier de Leyde (d'où le nom d'« éditions elzéviriennes »).

À la fin du XVIII ${ }^{\mathrm{e}}$, la contribution de la contrefaçon à l'aisance économique des imprimeurs bruxellois est telle que l'on voit apparaître une étonnante proposition : si les imprimeurs bruxellois unissaient leurs efforts, ils pourraient « s'emparer de presque tout le commerce de livres que font la Hollande, la France et l'Angleterre ". C'est un certain Derival qui écrit ces lignes. Elles sont peu connues, mais témoignent d'une bonne connaissance de la situation. Et pour une fois, il s'agit d'une opinion qui ne reflète pas un point de vue français sur la question :

26 Mémoires de Marmontel. Paris, Firmin-Didot, 1884, p. 365.

27 On l'a vu plus haut, Plantin déjà tentait de contrôler la reproduction de ses ouvrages par des imprimeurs situés en dehors des Pays-Bas espagnols. En fait, la contrefaçon naît quasiment avec l'imprimerie commerciale, au $\mathrm{Xv}^{\mathrm{e}}$ siècle. 
Il n'y a pas de ville en Europe mieux située que Bruxelles pour le commerce de libraire ; elle pourrait, si les imprimeurs étaient plus unis entre eux qu'ils ne le sont, s'emparer de presque tout le commerce de livres que font la Hollande, la France et l'Angleterre. Les imprimeurs de Bruxelles contrefaisant tous les bons livres qui s'imprimeraient à Paris, à Amsterdam ou à Londres, vendraient à moitié moins leurs contrefactions, que ne pourraient donner leurs livres les imprimeurs de ces trois villes ; ils auraient donc sur eux la préférence et feraient un bénéfice considérable, vu qu'ils n'auraient pas à payer de manuscrits et qu'ils paieraient les papiers et les caractères moins cher, ainsi que leurs ouvriers. Huit ou quinze jours après qu'un livre aurait paru à Paris, il pourrait être mis en vente à Bruxelles, où il serait contrefait : mais cela ne pourra se faire que lorsque les imprimeries de Bruxelles se réuniront et agiront de concert, ou qu'il s'établira dans cette ville une société typographique dont les fonds suffiront pour faire toujours rouler 12 à 15 presses $^{29}$.

L'importance économique de la contrefaçon ne prédispose sans doute guère les imprimeurs bruxellois à devenir des libraires, au sens où le $\mathrm{XVIII}^{\mathrm{e}}$ siècle français l'entend. Ils restent confortablement assis derrière leurs presses et reproduisent tout ce qui passe. Certains, cependant, vont prendre des risques. C'est ainsi que Jean-Louis de Boubers, pourtant installé dans l'exploitation des commandes d'État, va oser publier en 1774 les œuvres de Rousseau en douze volumes in $-4^{\circ}$, avec la mention « Londres » pour tromper les autorités (L'Émile était interdit aux Pays-Bas). Tout se passe alors comme si les réflexes commerciaux de l'imprimeur, qui sait faire des coups en matière d'ouvrages à contrefaire rapidement, s'étendaient aux œuvres politiquement interdites et l'amenaient à devenir un libraire-éditeur aux ambitions autant intellectuelles qu'économiques. Bien entendu, cette capacité à braver les interdictions peut tout aussi bien jouer en faveur de la reproduction de livres pornographiques. C'est ici qu'il faut revenir à Bassompierre, parce qu'il présente un beau cas de passage de l'acte du contrefacteur à l'acte éditorial. D'imprimeur vaguement licencieux, il devient, sous l'influence du journaliste français Pierre Rousseau, un des éditeurs liégeois des Encyclopédistes.

\section{Sens des affaires et sens du coup}

Il faut attendre la seconde moitié du Xvi ${ }^{\mathrm{e}}$ siècle pour trouver un imprimeur établi à demeure dans la Principauté de Liège. Cette étonnante carence est sans doute due plus à l'absence d'enseignement supérieur qu'à la hantise des Princes-Évêques de voir le protestantisme se répandre sur leurs terres. Gautier Morberius sera suivi d'autres imprimeurs-libraires, qui constitueront autant de dynasties familiales, notamment par les veuves. Au XVII ${ }^{\mathrm{e}}$, le retard est rattrapé, surtout du côté des traités religieux et des livres de piété ${ }^{30}$, mais les travaux historiques, juridiques et scientifiques ne sont pas totalement absents. Certes, les Princes-Évêques mettent en place dès la fin $\mathrm{du} \mathrm{XvI}^{\mathrm{e}}$ un lourd appareil de contrôle des productions. Mais les imprimeurs apprennent peu à peu à contourner les règlements et, au début du XVIII ${ }^{\mathrm{e}}$, ils sont passés maîtres dans l'art des fausses adresses et des publications sans lieu ni date. La contrefaçon d'ouvrages étrangers leur est donc toute naturelle ${ }^{31}$.

29 Derival, Le Voyageur dans les Pays-Bas autrichiens, ou lettres sur l'étact actuel de ces pays, 1782-1784 (cité par « De Rg. » dans Le Bibliophile belge, t.VI, 1849, p. 140). Le commentaire de ce «bibliophile belge » est éclairant : il fulmine contre la proposition de Derival, qui «n'a envisagé l'imprimerie et la librairie que sous le point de vue matériel, et a tourné le dos au point de vue moral ». Toutes ces informations proviennent du texte déjà cité de J. Brassine.

31 La méthode utilisée par certains imprimeurs liégeois du $\mathrm{xvII}^{\mathrm{e}}$, telle qu'elle est décrite par Pilati di Tassulo, n'est pas sans rappeler, par son cynisme tranquille, celle des imprimeurs lyonnais à l'égard des libraires anversois un siècle plus tôt. À Anvers au XvII : «La correspondance des Verdussen nous révèle sous ce rapport des procédés vraiment extraordinaires. Du moment qu'un libraire n'obtient pas d'un collègue étranger des conditions d'achat avantageuses pour une édition quelconque, immédiatement il annonce que, par mesure de représailles, il va faire réimprimer cette édition »-SABBE (M.), «La typographie anversoise... ", Histoire du livre et de l'imprimerie..., op.cit., t. IV, pp. 84-85. À Liège au xvI" siècle : «Les imprimeries de Liège et de Maestricht font un grand tort à celles de la Hollande parce que dans ces deux villes la liberté de la presse étant égale, et le prix de la main d'œuvre plus bas qu'en Hollande, les 
Tout au long de sa carrière, Bassompierre joue sur plusieurs tableaux. Très officiellement « Imprimeur du Conseil Privé » du Prince-Évêque, il est autorisé à « imprimer tous les mandements, réglements et édits qui émaneront de son autorité princière ${ }^{32}$. Mais, de diverses sources, il apparaît qu'il publie sous le manteau divers ouvrages légers tel le célèbre (et la délicieuse) Fanny Hill de John Cleland. Il se fait également le spécialiste des livres du marquis de Caraccioli, qui n'ont pas particulièrement bonne presse ${ }^{33}$. Mais son catalogue comprend par ailleurs des ouvrages de littérature féminine bien pensante et une multitude de livres très sages. Il n'y a donc pas, chez Bassompierre, de ligne éditoriale affirmée, juste une volonté toute commerciale de faire prospérer son entreprise. Mais quand Pierre Rousseau lui propose en 1759 d'imprimer, en contrefaçon, un texte de Voltaire, Le Précis de l'Ecclésiaste, il n'hésite apparemment pas à faire un coup, qui sans être d'une grande audace exige un certain cran, vu le parfum de soufre que dégage le nom du philosophe français ${ }^{34}$. Il remet d'ailleurs cela un peu plus tard, avec la publication du Précis du Cantique des cantiques ${ }^{35}$. Et pendant quelques années, jusqu'en 1764-1765, Bassompierre va publier du Voltaire. Ce n'est pas qu'il soit converti aux idées philosophiques (en fait, il publiera des travaux antiphilosophiques quelques années plus tard). C'est simplement qu'il y a vu une bonne opération à réaliser. Du moins a-t-il osé l'entreprendre et, de notre point de vue, c'est la disposition à l'action constituée par la pratique de la contrefaçon qui lui a permis d'agir de la sorte. Nous voyons donc dans la contrefaçon la deuxième naissance de l'édition belge. Une naissance lestée d'atouts et de handicaps.

\section{Le soleil noir de la contrefaçon}

Parmi les effets positifs de la contrefaçon, il faut non seulement suggérer cette capacité à faire des coups (un peu) audacieux, mais une capacité à envisager les relations d'affaires dans une perspective internationale. Les contrefacteurs liégeois (et bruxellois) du XVIII ${ }^{\mathrm{e}}$ siècle gardent en effet un œil attentif sur les publications françaises et hollandaises, et entretiennent des réseaux de vente qui vont de Londres à Berlin, de La Haye à Lisbonne. La contrefaçon va en outre amener les imprimeurs, tout particulièrement liégeois, à renforcer leurs compétences techniques et à augmenter leurs capacités de production au delà des niveaux atteints par la plupart des grandes villes étrangères. Pour contrefaire habilement un ouvrage, il faut savoir jongler avec les corps et les casses. Il faut donc des ouvriers très expérimentés. Liège sera avec Paris « comme la moelle osseuse du grand corps des imprimeries francophones, formant quantité de compagnons qui s'en allaient ensuite, au

libraires peuvent donner toutes sortes de livres à meilleur marché, et quand un libraire hollandais a fait imprimer un livre qui est recherché par le public, les libraires de Maestricht et de Liège écrivent aussitôt au libraire de Hollande et lui en demandent telle quantité d'exemplaires pour un tel prix qu'ils fixent euxmêmes, en lui déclarant très nettement qu'au cas où ils y trouvent à redire ou qu'ils trainent trop à leur en faire l'expédition, ils en donneront tout de suite une contrefaction eux-mêmes. " - Pilati DI TAssulo, Lettres sur la Hollande, 1780, cité par FrancotTe (H.), La Propagande des Encyclopédistes ..., op.cit., p. 95.

32 Cité par GAson (P.-M.), « De Rousseau aux méfaits des contrefacteurs : une correspondance de LebrunTondu avec la Maison Barde et Mauget », dans DrorXHE (D.) et al., Livres et Lumières au pays de Liège (1730-1830). Liège, Desoer, 1980, p. 186.

33 Droixhe (D.), « Voltaire et l'édition liégeoise jusqu'en 1765 », dans DroixHE (D.) et al., op.cit., p. 150.

34 La figure de Pierre Rousseau mériterait à elle seule une étude complète, car elle ressemble fort à celle d'un éditeur moderne. Né à Toulouse en 1716, Rousseau est à Paris de 1740 à 1755, où il se lie avec Voltaire, d'Alembert et Buffon. Il a l'idée de créer un journal qui diffuserait les idées des Lumières. Ce n'est pas possible à Paris. On lui suggère la Principauté de Liège, où la censure est molle et où les ouvriers typographiques sont de qualité. C'est ainsi que le Journal Encyclopédique sort le $1^{\text {er }}$ janvier 1756 des presses d'Everard Kints. Mais les théologiens de l'Université de Louvain et les chanoines de Liège se liguent contre P. Rousseau, qui s'enfuit en 1759 pour le duché de Bouillon. L'entreprise (ou le réseau) P. Rousseau est une véritable maison d'édition, qui a ses écrivains, ses presses, ses organes de diffusion. Vont en sortir pendant une trentaine d'années des journaux et des ouvrages de première importance pour l'histoire intellectuelle européenne; cf. BIRN (R.), «Le livre prohibé aux frontières : Bouillon », dans Histoire de l'édition française, op.cit., II, pp. 334-341.

35 La collaboration de Pierre Rousseau avec Bassompierre a été mise en lumière par DroIXHE (D.), art.cit., pp. 151-158. 
gré d'une constante circulation de main d'œuvre, compléter le personnel d'autres ateliers ${ }^{36}$. Pour contrefaire systématiquement, il faut aussi des presses nombreuses. Ces capacités de production sont attestées non seulement par des chiffres absolus mais encore par les associations, réalisées ou non, entre des imprimeurs liégeois et des industriels de la culture comme Charles-Joseph Panckoucke. On sait en effet qu'en 1778 une édition complète de l'Encyclopédie en 36 volumes a été proposée aux Bassompierre (père et fils). Le projet n'aboutit pas mais il témoigne d'un fait : « les Bassompierre [...] étaient capables d'assumer la charge financière et technologique d'un ouvrage tel que l'Encyclopédie » ${ }^{37}$. Quelques années plus tard, Panckoucke, qu'un contemporain appelait le « marchand d'idées » ${ }^{38}$, propose à l'imprimeur liégeois Clément Plomteux de sortir une énorme Encyclopédie méthodique, offerte en souscription ${ }^{39}$. Revirement de Panckoucke : l'association tourne court après quelques années (et quelques volumes). Mais peu importe : elle montre que Plomteux se sent les reins assez solides pour entreprendre des projets d'une ambition apparemment démesurée pour l'époque. Et dans son cas, il ne s'agit pas seulement de compétences techniques : il injecte des capitaux.

Reste, comme le fait remarquer Gossiaux, que «c'est évidemment dans la conception et l'élaboration scientifique de l'ouvrage [l'Encyclopédie] que la participation des Liégeois est la plus faible " ${ }^{40}$. Le premier, le plus grave des effets désastreux de la contrefaçon apparaît ici : la France produit et la Belgique reproduit, la
France crée et la Belgique diffuse. La contrefaçon va empêcher la naissance de l'édition moderne en Belgique au début du $\mathrm{XIX}^{\mathrm{e}}$ siècle, parce qu'elle va inhiber les entreprises éditoriales intellectuellement créatrices et novatrices. Alors qu'en France les imprimeurs vont se recroqueviller et laisser peu à peu la place à des éditeurs qui seront à la fois des hommes d'art et d'argent, des industriels et des intellectuels, on va assister en Belgique, de 1800 à 1840, à un épanouissement de l'imprimerie «brute » grâce à la contrefaçon industrielle. On peut en effet formuler l'hypothèse que la contrefaçon mise en place à Liège et à Bruxelles, à une échelle quasi industrielle dès la fin du $\mathrm{XVII}^{\mathrm{e}}$, favorise l'éclosion précoce de réflexes économiques et de structures de production puissantes qui vont permettre à un Henri Casterman, par exemple, de devenir rapidement un éditeur complet. Si du moins l'on admet de voir dans l'impression à la chaîne de livres religieux un travail éditorial créatif.

\section{III. \\ Casterman ou les temps bénis de l'édition religieuse}

Les imprimeurs bruxellois et liégeois pénètrent dans le $\mathrm{XIX}^{\mathrm{e}}$ siècle bien équipés, techniquement et financièrement ${ }^{41}$. Après quelques années difficiles sous le régime napoléonien, beaucoup plus sévère à leur égard, ils vont s'épanouir en tous sens jusqu'en 1854, année de mise en application de la Convention passée en 1862 entre la France et la Belgique, qui met fin à la contrefaçon. Mais pendant près d'un demi-siè-

36 RYChNer (J.), « À l'ombre des Lumières : coup d'œil sur la main d'œuvre de quelques imprimeries du XvIII ${ }^{\mathrm{e}}$ siècle ", cité par GASON (P.M.), art.cit., p. 186, note 5.

37 Gossiaux (P.P.), « L'encyclopédie liégeoise (1778-1792) », dans Droixhe (D.) et al., op. cit., p. 201.

38 Cité par Tucoo-Chala (S.), Charles-Joseph Panckoucke \& la Librairie française 1736-1798. Paris, Marrimponey Jeune-Jean Touzot, 1977, p. 129.

39 En 49 volumes in- $4^{\circ}$, soit en 91 volumes in- $8^{\circ}$; cf. Gossiaux (P.P.), art.cit., pp. 205-217.

40 Gossiaux (P.P.), art.cit., p. 220.

41 Le regard extérieur que l'éditeur Poulet-Malassis, durant son bref exil en Belgique, portera en 1863 sur le secteur éditorial local n'est pas sans enseignement et peut être apporté à l'appui de notre hypothèse conductrice. S'il se plaindra très vite, à la façon de son ami Baudelaire, de ce qu'il appelle «l'hébétude belge ", Poulet-Malassis ne manquera pas en effet de saluer le savoir-faire des imprimeurs bruxellois : « On y imprime à la presse à bras et très bien, car il y a encore une population considérable d'ours élevés dans les traditions. [...] J'ai été très surpris de voir que cette contrefaçon très vilipendée était bien supérieure à la production parisienne originale. Il y a notamment toute une collection de poètes modernes à bas prix, en format minuscule, qui est une chose excellente dans son genre, et toutes sortes de romans à 50 centimes le volume qui sont des chefs-d'œuvre (vous le croirez sans peine), relativement à la collection Lévy » — Lettre à Charles Asselineau, 26 octobre 1863, citée par Pichors (Cl.), Auguste Poulet-Malassis. L'éditeur de Baudelaire. Paris, Fayard, 1996, p. 170. 
cle, ce sera le règne du libéralisme le plus explicite ${ }^{42}$. À partir de 1814 , toute personne exerçant une activité d'imprimerie a le droit d'imprimer, éditer, vendre les ouvrages qui ne sont pas l'œuvre d'un auteur résidant en Belgique et en Hollande. Il n'y a donc aucune législation internationale concernant le droit d'auteur. En outre, Guillaume $\mathrm{I}^{\mathrm{er}}$ stimule une politique à l'exportation. Sur le subside annuel alloué par l'État à l'industrie, il assigne des primes destinées à encourager les éditeurs à expédier leurs produits à l'étranger ${ }^{43}$. Grâce à ces mesures, l'exportation prend de l'importance. On ouvre des « Comptoirs de librairie » à Londres et à Leipzig ${ }^{44}$. Combinée au fait que la Restauration impose à la France une censure sévère et que le prix des livres y est trop élevé, cette attitude du gouvernement hollandais va faire exploser les industries graphiques bruxelloises.

L'indépendance belge perturbe cette belle envolée. La Belgique perd son marché le plus important. En effet, la Hollande oppose un blocus à tous les produits des provinces du sud des Pays-Bas. La situation du livre en Belgique aurait pu devenir catastrophique si la librairie française avait réagi. Au contraire, elle continue à élever ses prix et la contrefaçon belge peut continuer d'écouler ses produits. Un second obstacle aurait pu briser l'élan : le manque d'organisation interne. La concurrence est si sauvage entre petits imprimeurs qu'ils se cassent mutuellement le marché. La crise de 1830 va « nettoyer » celui-ci. Les trop petites imprimeries et librairies disparaissent, de grandes associations se forment. Cinq grandes sociétés se constituent sous le patronage de la Banque de Belgique ${ }^{45}$. Ce sont les débuts du capitalisme d'édition ou plutôt d'imprimerie, de librairie et d'édition, car il n'y a pas de capitaux spécifi- quement placés en chacune de ces activités, qui resteront encore longtemps confondues. Mais le fait essentiel est que le monde des affaires prend conscience de l'importance économique du marché de la diffusion du savoir. Avec la constitution de ces nouvelles sociétés, on voit arriver au sein des conseils d'administration un nouvel actionnariat : «l'on remarque », note ainsi Charles Hen (contemporain de cette mutation), « beaucoup d'hommes distingués par leur haute position ou par leur rang social, des banquiers, des magistrats, des représentants, des sénateurs ${ }^{46}$.

\section{Habitus techniciste et éthos reproducteur}

Pendant ces années de contrefaçon systématique, tout passe sous les presses belges : l'abondante production romantique, la littérature juridique et scientifique, les partitions de musique et jusqu'aux revues, telle La Revue des deux Mondes. Sous la pression de la concurrence, les imprimeurs produisent dans tous les formats et à des prix de moitié inférieurs à ceux de Paris. En 1834, Stendhal écrit à Sainte-Beuve : « Rome et moi, nous ne connaissons la littérature française que par l'édition belge ${ }^{47}$. Il va sans dire que la France organise la lutte contre la contrefaçon. Tout d'abord, les contrefaçons belges de livres français sont légalement interdites en France. La douane française exerce une surveillance sévère et les éditions originales de livres belges en subissent les conséquences. Il faut joindre à l'envoi un certificat sur timbre de l'origine de son livre, déclaration signée par le bourgmestre de sa municipalité. Des envois sont examinés à la frontière, avec possibilité de saisie. «Un droit d'entrée de $100 \%$ est perçu sur toute publication belge qui entre en France, alors

42 Les sources principales de cette synthèse sont classiques : LIEBRECHT (H.), Le Livre et l' Imprimé en Belgique durant la première moitié du xIX ${ }^{\ell}$ siècle. Bruxelles, Musée du livre, 1934 ; DopP (H.), La Contrefaçon des livres français en Belgique. Louvain, Vuystpruyst, 1932. Un mémoire de licence sur les pratiques de contrefaçon a d'autre part été réalisé, à l'Université de Liège, sous la direction de Jean-Marie Klinkenberg, par Olivier LedAIN : La Contrefaçon dans la librairie en Belgique au début du xIxésiècle et ses répercussions sur les littératures belges et françaises. Département d'études romanes, 1995. HeN (Ch.), La Réimpression. Bruxelles, 1851, p. 43.

Ibid., p. 44.

45 La Société typographique belge, Ad. Wahlen et comp., capital social : $1.000 .000 \mathrm{~F}$; la Société belge de librairie, imprimerie et papeterie, capital social : $1.500 .000 \mathrm{~F}$; la Société de libraire, imprimerie et fonderie, capital social : $2.000 .000 \mathrm{~F}$; la Société encyclographique, capital social : $1.000 .000 \mathrm{~F}$; cf. HEN (Ch.), op.cit., pp. 48-49.

47 Deschamps (J.), « Stendhal et la Belgique », cité par Dopp (H.), op.cit., p. 21. 
que les livres français ne payent que $30 \%$ de droit d'entrée en Belgique " ${ }^{48}$. Ensuite, les libraires de Paris accusent les imprimeurs belges de leur fermer tout débouché hors de France par des prix bradés. Dopp a montré que l'exportation française est restée 5 à 10 fois plus importante que l'exportation belge ${ }^{49}$. De plus, ainsi que certains observateurs le soulignent déjà à l'époque (l'éditeur Hetzel notamment), les imprimeurs belges ont ouvert par leur pugnacité et leurs prix de nouveaux marchés étrangers aux libraires français. Enfin, certains écrivains français se déchaînent contre « cette damnée Belgique », ainsi que Balzac l'appelle, qui avec Jules Janin vitupère contre la contrefaçon belge, laquelle « [vole] aux écrivains français la meilleure partie de leurs revenus ».

En réalité, il faut selon Dopp distinguer entre deux catégories d'écrivains. Les plus lus à l'époque (Balzac, Musset, Hugo, Vigny, Janin, Nodier, Sand, Mérimée, Béranger) s'élèvent contre la réimpression belge parce qu'ils veulent protéger les éditeurs français avec qui ils passent des contrats d'exclusivité. Les autres, les obscurs, les sans grades, ont au contraire tout intérêt à se faire piller, car les tirages belges leur apportent la renommée ( Quand on voulait assurer le succès d'un livre, on annonçait officieusement qu'il était déjà contrefait en Belgique »). Nos éditeurs belges vont d'ailleurs délaisser les premiers, qui prêtent à tellement de concurrence que les prix de vente écrasés ne laissent que de faibles bénéfices, au profit des seconds, romanciers feuilletonistes ou faiseurs de vers. Bien sûr, ces imprimeurs lisent peu, n'ont guère d'esprit critique et se laissent parfois éblouir par les produits venant de Paris. Cependant, la contrefaçon ne produit pas que des livres intellectuellement et graphiquement médiocres. Au contraire, elle pousse parfois les imprimeurs bruxellois à réaliser des performances étonnantes, comme en témoigne un écrivain anglais, contemporain de Hen :

Sur les marchés extérieurs, on a cessé de voir et ces honteuses pages blanches, et ces scandaleuses interlignes qui chassent le texte, et ces insolentes marges qui, dans la plupart des livres français, dévorent l'espace aux dépens de la pensée. Les éditions belges, réduites à de justes proportions, dégagées de toutes les sophistications dont les éditeurs parisiens les plus candides ne savent pas toujours se défendre, sont venues s'offrir aux acquéreurs à 50,60 et $70 \%$ de rabais, et souvent avec 12 et 14 mois de terme. Aussi lorsque à Londres je vis tout à coup étaler, dans Fleet street, sur le Strand, à Soho-square, dans Regent-street et à Burlington-Arcade, ces magnifiques volumes à deux colonnes, dans le format de nos imperial octavo, avec les noms de Lamartine, de Casimir Delavigne, d'Alexandre Dumas, de Victor Hugo, je crus un instant que la librairie parisienne avait transporté ses comptoirs et ses ateliers à Bruxelles. Point du tout : CE N'ÉTAIENT QUE DES CONTREFAÇONS SUPÉRIEURES AUX ÉDITIONS ORIGINALES $^{50}$.

Aux alentours de 1845, la contrefaçon amorce un déclin rapide. Concurrencées par des éditeurs industriels français (Mame, notamment) qui parviennent à vendre en France et en Belgique des ouvrages de grande qualité technique à des prix très bas, affaiblies par une course folle au texte et à la baisse des prix, les grandes sociétés belges de librairies disparaissent en quelques années (1846-1851). Dans un mouvement dont est coutumier le champ économique, ne parviennent à subsister que de petits imprimeurs, dont certains réussissent même à s'imposer parmi les éditeurs les plus importants du temps. Ceux-ci se concentrent à Bruxelles. De plus, l'opinion publique qui, au xvm ${ }^{\mathrm{e}}$ et au début du $\mathrm{XIX}^{\mathrm{e}}$ siècle, acceptait parfaitement cette industrie, se retourne contre elle, tenue désormais pour malhonnête et immorale. La Société des Gens de Lettres adresse en 1849 une pétition à la Chambre et demande la suppression de la contrefaçon. On y relève la phrase suivante, lourde de sens et de bon-sens : « abolir la contrefaçon, c'est créer la littérature belge, c'est lui permettre d'exister ${ }^{51}$.

48 Dopp (H.), op.cit., p. 88.

49 Dopp (H.), op.cit., p. 93 sq.

50 Cité d'après Hen (Ch.), op.cit., pp. 48-49.

51 Dopp fournit une explication plus complète : «Ce n'étaient point les Meline, les Hauman, qui pouvaient songer à acquérir des manuscrits belges, à offrir une rémunération à des auteurs encore sans titres et sans notoriété : ils trouvaient sans frais, dans la littérature française, matière à des réimpressions d'une vente 
L'État en semble conscient : il crée en 1848 un Institut National des Lettres belges, institue une Commission pour l'encouragement de la littérature et de l'art dramatique et se charge de payer des primes aux représentations d'auteurs dramatiques belges. Il hésite cependant à interdire la contrefaçon: les libéraux la protègent, les catholiques veulent la supprimer. La France lui fournit l'occasion d'une décision en rangeant la suppression parmi les conditions fondamentales du renouvellement du traité de commerce de 1852. Une convention est signée le 22 août 1852, approuvée par la loi belge du 12 avril 1854 et mise en vigueur le 12 mai 1854. L'application de cette convention n'apaisera pas les éditeurs français. On pourra lire dans le Rapport du Jury de l'Exposition Universelle de Paris de 1855 les propos suivants :

Les productions de la Belgique appartiennent à l'École française. Les publications des imprimeurs belges ont, en effet, avec les nôtres un air de famille qui ne surprend pas et qui a sa cause naturelle dans l'ancien commerce de reproduction de nos ouvrages et dans l'usage de la même langue. Plusieurs imprimeurs fabriquent des volumes qui pourront un jour lutter avec les nôtres pour l'exécution typographique. Nous devons y faire attention, alors que nos confrères bruxellois ont manifesté l'intention de venir nous faire concurrence sur le marché de Paris ${ }^{52}$.

À cette Exposition, douze maisons belges sont représentées. En chiffres, les exportations belges sont évaluées à 500.000 francs français et l'essentiel de la production est composée de livres en langues mortes ou étrangères et d'ouvrages à caractère liturgique.

\section{Les secours de l'Église}

Ce dernier point important. Il explique les raisons pour lesquelles la production du livre en Belgique ne s'est pas écroulée après 1854 .
Car les chiffres disponibles montrent que le passage à l'après-contrefaçon se fait sans crise grave. Un document officiel produit pour l'Exposition Universelle de 1870 montre que la production s'est rapidement réorientée « vers les éditions originales et les ouvrages du domaine public destinés au marché intérieur " ${ }^{53}$. Parmi ces ouvrages du domaine public, il faut sans doute placer les ouvrages scolaires, les livres de prix - et les livres religieux. Ceux-ci, en outre, s'exportent. Et d'après les savants relevés de Claude Savart, on peut même dire qu'il s'exportent en quantités industrielles ${ }^{54}$. Savart montre d'une part que les importations de livres (en tonnes) en provenance de Belgique ne cessent d'augmenter depuis le milieu du siècle, passant de 119,5 tonnes en 1851 à 205,7 en 1896, et d'autre part que le pourcentage de titres religieux belges mentionnés dans la Bibliographie de la France passe de $0 \%$ en 1853 à $41 \%$ en 1859.

On avancera donc que l'édition religieuse a sauvé le commerce du livre en Belgique après l'arrêt de la contrefaçon. Mais pourquoi, de tous les genres éditoriaux possibles, le livre religieux a-t-il connu cet essor ? Sans doute grâce à la conjonction de deux facteurs. D'une part, la contrefaçon elle-même y a contribué de plusieurs manières : elle a formé en Belgique des typographes extrêmement qualifiés, ceux-là mêmes qu'exigent la composition et l'impression de livres religieux ${ }^{55}$. Plus globalement, elle a installé en Belgique une disposition à la pratique industrielle et au commerce international du livre et, comme on l'a vu plus haut, une capacité à reproduire fidèlement un texte venu d'ailleurs - non plus en l'occurrence de Paris, mais de Rome. Ce n'est donc pas que l'on assiste en Belgique à la conversion d'anciens contrefacteurs en imprimeurs religieux. Ce seront en fait d'autres éditeurs qui vont se développer (Casterman, Dessain, Wesmaël-Legros, Desclée). Mais la contrefaçon, du $\mathrm{Xv}^{\mathrm{e}}$ à la première moitié du XIX ${ }^{\mathrm{e}}$, a construit l'espace mental des possibilités éditoriales du pays. Et le livre religieux de la deuxième moitié du $\mathrm{XIX}^{\mathrm{e}}$ va

mieux assurée. Et pour nos auteurs, il était à peu près impossible, même en renonçant à tout profit, de conquérir une place en librairie, au milieu de la masse des contrefaçons " (op. cit., p. 179).

Cité par LieBrecht (H.), Histoire du Livre..., V, p. 105.

Statistique de la typographie en Belgique, dans Le Bibliophile belge, 1870, p. 33.

SAVART (Cl.), Les catholiques en France au XIX ${ }^{e}$ siècle : le témoignage du livre religieux. Paris, Beauchesne, 1985 , pp. 82-83.

Anonyme, cité par SAVART, ibid., pp. 82 et 84 . 
répondre exactement aux dispositions qu'elle a peu à peu façonnées. C'est ici qu'intervient le second facteur. Sous l'impulsion de Léon XIII, les études exégétiques, les recherches thomistes, les remises à jour (notamment du chant grégorien) connaissent un essor international. En Belgique, l'Abbaye du Mont César, près de Louvain, l'Université Catholique de Louvain et quelques autres centres religieux sont à la tête de ce renouveau liturgique. Ils vont faire publier leurs recherches chez des imprimeurs belges existants (Casterman, Brepols) ou chez des industriels dont ils soutiennent la reconversion en imprimeurs de haute technicité ${ }^{56}$. Ils leur confèrent des monopoles de vente sous la forme du titre d' "Imprimeur du Saint Siège » (titre assez facilement accordé : il y en avait quatre ou cinq en Belgique) et d' « Imprimeur de la Sainte Congrégation des Rites » (titre très rare : huit éditeurs portaient ce titre dans le monde, dont deux en Belgique, Desclée-Tournai et Dessain-Malines). Le marché religieux est énorme : outre le fait qu'il est mondial, puisque ses produits sont écrits uniquement en latin, la littérature liturgique, patrologique, exégétique remplit des milliers de pages ${ }^{57}$.

En étudiant la montée de la dynastie Casterman, on peut voir comment s'accomplit cette conjonction d'éléments apparemment disparates. On peut voir aussi comment s'accomplit l'émergence progressive, sur trois générations, d'un éditeur au sens plein du terme. C'est la troisième naissance de l'édition belge.

\section{3. "Pro Deo et Patria "}

L'histoire du fondateur, Donat Casterman, se lit comme un récit d'édification morale ${ }^{58}$. Orphelin très jeune, il entre en apprentissage chez un peintre, F.-J. Manisfels, puis chez un imprimeur, Nicolas Jovenau, qui l'initie à la reliure et au commerce de la librairie. Et tel un Plantin des temps modernes, il s'installe libraireéditeur à 24 ans, en août 1780. Les activités éditoriales sont très modestes : elles se limitent à faire imprimer à son nom de petits livres scolaires et religieux pour le marché local. On voit ainsi apparaître en 1794 un Petit Catéchisme des Catholiques en faveur des jeunes écoliers. Sous le titre, Donat Casterman se présente comme « libraire et relieur» - non comme « imprimeur » et moins encore comme « éditeur» 59 . Imprimeur, il le devient en fait en 1803, après avoir acheté trois maisons rue des Tanneurs. Il reçoit son brevet d'imprimeur en 1804 et devient «Imprimeur de l'Évêché de Tournai », nouvellement établi, en 1805. Cette double installation officielle va définitivement lancer l'imprimerie Casterman (qui jouira d'un rapport privilégié avec l'Église jusqu'au début des années 1960). Dès 1806, Donat Casterman présente un catalogue de productions maison. On n'y trouve pas (et les catalogues des années ultérieures ne démentent pas cette analyse) la trace d'une activité éditoriale originale. Il s'agit de livres à compte d'auteur, d'ouvrages religieux, de manuels scolaires. Donat Casterman imprime

56 Dans les années 1870-1880, le Primat de Belgique, le Cardinal Deschamps, incite les deux frères Desclée à fonder une imprimerie à Tournai et une imprimerie à Bruges - qui deviendra plus tard la maison Desclée de Brouwer - , ce qu'ils réalisent en faisant venir d'Angleterre des presses perfectionnées et des ouvriers typographes très spécialisés.

57 «La liste officielle des livres liturgiques en usage dans l’Église romaine (décret de la Congrégation des Rites, 17 mai 1911) comprend essentiellement : le Bréviaire, le missel, le Rituel, le Pontifical, le Martyrologe, le Cérémonial, les Propres des offices et messes, le Memoriale Rituum, l'Instruction clémentine pour l'exposition du Saint-Sacrement, la Collection des décrets de la Congrégation des Rites, les livres de chant grégorien, le Code de droit canonique » (Grand Larousse Encyclopédique, t. 6, p. 795).

58 Les données historiques de cette reconstitution sont puisées à deux sources. Une source officielle, assez lacunaire mais précieuse pour ses reproductions d'archives : Casterman: deux cents ans d'édition et d'imprimerie. Tournai, Casterman, 1980. Une source officieuse, plus précise par moments (notamment quant aux rapports de Casterman avec l'Église) : Caspaton (journal d'entreprise de Casterman), 2 (Noël 1971), 3 (Pâques 1972), 4 (Juillet 1972), 5 (Noël 1972). Pour plus d'informations à caractère historique et catalographique, on se reportera à la monographie publiée par Serge BoufFange: Pro deo et patria. Casterman : Librairie, imprimerie, édition (1776-1919). Genève, Droz, 1996.

59 Comme on l'a vu plus haut, le terme n'apparaît dans son acception moderne qu'au milieu du XIX ${ }^{\mathrm{e}}$ siècle. De fait, c'est sur un ouvrage de 1845 que J(osué) Casterman mentionne pour la première fois le titre « libraire-éditeur ». 
(ou réimprime), relie et vend sur place, dans sa propre librairie. La maison Casterman est donc fondée sur le commerce de livres "préfabriqués ", c'est-à-dire de livres dont le processus d'élaboration intellectuelle échappe totalement à leur « éditeur ».

Très tôt, Donat Casterman associe ses fils à son entreprise. Josué, l'aîné, s'occupe de la librairie dès 1808 (il a 25 ans), tandis que le fils cadet, Charles, reprend peu à peu la direction de l'imprimerie (à partir de 1814 - il n'a que 17 ans). Quand le père se retire en 1820 , il lègue à ses fils un fonds de quelques dizaines d'ouvrages et une imprimerie dotée de cinq presses autour desquelles s'activent une vingtaine de personnes. Mettant alors en place une structure de division du travail qui se perpétuera jusqu'à nos jours, les deux frères vont faire évoluer les deux branches d'activité de l'entreprise Casterman de manière autonome mais coordonnée. Josué reste libraire et devient éditeur. Il reprend une partie du fonds paternel et l'augmente de ses titres propres. Charles continue de diriger l'imprimerie (avec un commanditaire appelé, ça ne s'invente pas, Dieu) et reprend la charge d'Imprimeur de l'Évêché (notamment le célèbre Grand double Almanach dit de Liège). Ces activités parallèles n'empêchent pas les deux frères d'avoir des activités croisées. Josué imprime tandis que Charles édite (seul ou avec son frère), ce qui donne des livres griffés « Chez J. Casterman, Fils Aîné, Libraire », « Chez Ch. Casterman-Dieu, Imprimeur-Libraire » ou « Chez les deux Casterman ». Une importante coproduction des deux frères est ainsi la Bibliothèque de la Jeunesse Chrétienne, en douze volumes. Par ailleurs, Josué lance en 1827 Le Courrier Tournaisien, qui deviendra Le Courrier de l'Escaut en 1829 en changeant d'imprimeur. Visiblement les affaires prennent de l'extension.

La mort de Charles en 1829 ne freine apparemment pas les activités de Josué, qui devient à son tour « Imprimeur de l'Évêché ». Il va faire agrandir l'imprimerie à quatre reprises entre 1834 et 1840 . Un catalogue de 1844 propose 250 titres qui n'appartiennent plus seulement aux domaines scolaires et religieux. Les rubriques du catalogue font place aux « littérature, sciences, histoire, éducation, aventures et voyages ». C'est vers 1844, précisément, que Josué,
« type [même] du bourgeois conquérant " ${ }^{60}$, qui ouvrira une première succursale à Paris, va inscrire sur la page de couverture de ses livres la dénomination « imprimeur-libraire-éditeur » ou encore celle de « libraire-éditeur ». L'apparition du terme «éditeur » et la disparition progressive du terme "imprimeur » (achevée vers 1855) reflètent sans doute à la fois l'évolution éditoriale générale et l'« affinement " particulier de l'imprimerie Casterman qui, vraisemblablement, cherche peu à peu à se présenter sous l'aspect plus « noble » d'une maison d'édition, dotée d'un réseau de vente international. Mais il ne faut pas s'y tromper. Si l'éventail du catalogue s'élargit, les plus gros chiffres de vente continuent à provenir de l'impression de petites brochures populaires (tels les billets des Rois, lancés par Donat Casterman vers 1805-1810, ou le Grand double Almanach qui tire à plus de 100.000 exemplaires par an). Une autre production à grande échelle est constituée par les livres de prix, qui apparaissent vers 1838 et qui vont bientôt sortir au rythme étonnant de 50 nouveautés par an. Des catalogues spéciaux de livres de prix seront envoyés dans toutes les écoles catholiques (avec la mention « Imprimeur de l'Évêché »). Ce sont là des productions éditorialement très simples (mais graphiquement très réussies), très prévisibles, très répétitives, qui ne sont pas sans faire songer aux futures locomotives de la maison Casterman : les annuaires téléphoniques et les albums de Tintin. Une fois composées, ces productions ne demandent en général que de minimes modifications avant d'être tirées à nouveau en X milliers d'exemplaires. Mais n'anticipons pas.

En 1849, Josué associe officiellement ses fils Henri et Alexandre à l'entreprise en changeant la dénomination en « $\mathrm{J}$. Casterman et fils ». Il se retire définitivement des affaires en 1856 et Henri redéploie toutes les activités de la maison. Sur le plan local, il va moderniser l'imprimerie en l'installant dans de nouveaux locaux et en la dotant de machines à vapeur. C'est ainsi qu'en 1863, 145 ouvriers et 36 ouvrières travaillent dans les ateliers. Le catalogue de cette même année comporte 1.500 ouvrages répartis en 65 rubriques et sous-rubriques. Sur le plan international, Henri crée une succursale à Paris et met au point un accord de diffusion réciproque avec la firme Kittler de 
Leipzig. C'est donc à la troisième génération de Casterman que l'on voit apparaître un entrepreneur qui semble être autant un imprimeur qu'un éditeur. Et cette apparition est fondée non tant sur le redéploiement international ou sur les initiatives de Henri en matière de services de presse, de dépôts et d'offices, que sur un catalogue particulièrement épais. En effet, le catalogue de 1863 est énorme, en raison de la place très importante qu'y prennent les livres religieux. Les rubriques qui leur sont consacrées en témoignent : " sciences sacrées », « histoire ecclésiastique », « dévotion », etc. À ces pieux ouvrages s'ajoutent une multitude de livres édifiants destinés à la jeunesse (livres de prix, livres de bibliothèques populaires, etc.). Les titres des collections qui les regroupent traduisent bien cette volonté d'éducation morale, qui se double souvent d'un souci d'éducation civique : «Récits historiques belges », « Récits moraux et amusants », « Musée moral et littéraire de la famille ", « Récits historiques et légendaires ».

Rien là de très créatif, éditorialement s'entend, et l'on pourrait aisément reléguer Henri Casterman au rang d'imprimeur industriel. Mais ce serait oublier une dimension de l'édition professionnelle : la capacité à tenir sur un marché, à occuper un créneau et à en faire une sorte de vente de monopole. Il ne suffit pas de faire des coups, brillants mais sans lendemain, comme nombre d'éditeurs à vocation littéraire (incarnés par Curmer en Fance, par Deman en Belgique) le feront tout au long du siècle. Il faut encore savoir anticiper et planifier à long terme. La pratique de l'édition exige la compétence prévisionnelle : c'est celle qu'ont démontrée Hachette, Larousse ou le second Hetzel. Certes, ils n'ont pas édité de grandes œuvres, mais l'édition ne peut être réduite au seul secteur de la haute littérature. C'est pourquoi le catalogue 1863 de Henri Casterman nous paraît refléter l'émergence d'un éditeur nouveau en Belgique. Encore fort mâtiné d'imprimerie, mais presque prêt à s'en affranchir pour faire surgir la création de la contrainte. Son flair d'éditeur passe par cette prise en charge pleine et entière de l'édition religieuse et de ses succédanés pour la jeunesse et les écoles catholiques. Il occupe le créneau et l'exploite systématiquement ${ }^{61}$ : c'est de la politique éditoriale moderne.

Henri Casterman meurt en 1869 , juste avant de recevoir le titre d' "éditeur pontifical ». La maison va alors entrer comme en hibernation. Sans doute ses deux fils, Henri et Louis, vontils reprendre la direction en 1880 , mais la production ne s'adapte pas à la réalité du jour. La situation se dégrade encore vers 1900 . Et ce n'est que lorsque les deux fils de Louis arrivent aux affaires, en 1920, que celles-ci reprennent. Bientôt viendront les annuaires (à partir de 1926) et Tintin (à partir de 1934).

\section{IV. La chute d'Icare : essor et faillite de l'éditeur artiste}

On vient de le voir : après 1854 , certains imprimeurs belges, qui disposent d'un équipement typographique sophistiqué, vont nouer des liens étroits avec l'Église et exploiter le créneau des ouvrages religieux, particulièrement rentable vu le caractère mondial du marché. D'autres maisons, principalement bruxelloises, vont quant à elles s'orienter vers d'autres domaines, recouvrant ceux au sein desquels la représentation lettrée confine le métier d'éditeur. Attachées à la haute littérature et, pour certaines, aux esthétiques d'avant-garde, elles vont tirer parti des censures qui s'exercent en France et des contraintes économiques qui accablent, à la fin du siècle, la librairie française. Véritable terre d'asile pour opposants, proscrits, révolutionnaires et réfugiés de tout poil, la Belgique, située au carrefour des diverses cultures européennes, va voir se développer sur son territoire une intense activité intellectuelle. Les exilés sont en effet souvent à la tête de journaux, de groupements politiques ou philosophiques, organisent des conférences, écrivent des livres. Quelques maisons vont jouer ici un rôle de première importance pour l'impression et la diffusion de ces écrits. Le Second Empire et les débuts de la Troisième République couvrent une période durant laquelle quelques éditeurs belges ou établis en Belgique vont, à la faveur de circonstances politiques, juridiques, économiques et culturelles, inverser le mouvement traditionnel conduisant

$61 \mathrm{Cl}$. Savart a montré que «surtout de 1850 à 1880, l'édition religieuse connaît en France une singulière activité » et que l'apport de Casterman à celle-ci est loin d'être négligeable, notamment en matière de livres de piété et de dévotion («Le livre religieux », dans Histoire de l'édition française, op.cit., t. III, p. 403). 
les auteurs belges à se faire éditer en France. Et que cette période corresponde aux premiers balbutiements d'une littérature nationale ne laisse pas d'être significatif.

\section{Lacroix \& Verboeckhoven}

La toute jeune « Librairie A. Lacroix, Verboeckhoven et $C^{\text {ie }}$ » est une de ces maisons. Éditeurs attitrés de Victor Hugo après que celui-ci a pris le chemin de l'exil suite au coup d'État du Deux-Décembre, Lacroix \& Verboeckhoven réalisent un grand coup dès 1862 en publiant à grand bruit Les Misérables. L'ampleur de l'œuvre et les conditions strictes imposées par le poète (versement comptant d'une somme élevée et cession temporaire des droits) ne vont pas décourager les éditeurs qui, pressentant le succès, vont éditer sous leur double griffe un manuscrit dont chacun mesure l'importance à la fois littéraire et sociale. Le coup est porteur, et ouvre la porte de la maison à de jeunes écrivains débutants ou marginaux captés par l'exemple des grands maîtres ayant sauté le pas vers l'édition belge. C'est le cas de Ducasse dont Lacroix \& Verboeckhoven, sept ans plus tard, éditeront - à compte d'auteur - Les Chants de Maldoror (que Max Waller, l'un des premiers, saluera dans La Jeune Belgique). Deux ans auparavant, en 1867, Lacroix \& Verboeckhoven faisaient paraître l'un des grands textes fondateurs de la littérature belge, La Légende d'Ulenspiegel de Charles De Coster. Si l'entreprise s'est soldée par un échec (l'accueil du public belge, plutôt conformiste, reste froid et la critique ne fait guère écho à la sortie du livre), elle mérite d'être fortement soulignée : c'est la première fois en effet qu'un éditeur important tente de donner une impulsion à une création littéraire belge engourdie. C'est la première fois aussi que s'opère en Belgique la rencontre non fortuite sur l'étal des libraires, quand bien même reste-t-elle sans grands effets dans l'immédiat, de l'ambition commerciale et d'un projet culturel.

La maison, dont la renommée grandit parallèlement à celle de Hugo, son auteur vedette, a connu son apogée en 1862 avec le banquet des Misérables organisé à l'occasion de la sor- tie du roman et auquel sont invités tous les grands noms de la politique et de la littérature. Elle va cependant, dans les années qui suivent, perdre assez vite la place importante qu'elle occupe sur le marché tant national qu'international. Parmi les divers facteurs qui vont précipiter son déclin puis sa faillite, un manque de discernement éditorial (la maison a ainsi repris les fonds provenant de la contrefaçon d'une série d'autres sociétés créées avant 1852) se conjugue à une mauvaise gestion commerciale. Les événements politiques ont eux aussi leur importance. Soucieux de percer sur les marchés étrangers, Lacroix \& Verboeckhoven ouvrent des comptoirs à Livourne, Paris et Leipzig. Trop tôt ou trop tard : le commerce avec la France et l'Allemagne va être réduit à néant du fait de la guerre de 1870. C'est cependant sa tendance au gigantisme qui va avoir raison de l'entreprise : installée Avenue de la Toison d'Or, elle se dote d'immenses ateliers et entrepôts avec plusieurs ascenseurs ainsi qu'un petit chemin de fer pour le transport des livres. Infrastructure barnumesque pour une politique éditoriale qui patine. «Les frais de bureau et de loyer », comme le souligne Josse Sacré dans la notice qu'il consacre à la maison, vont " [engloutir] toutes les espérances » ${ }^{62}$. La machine s'écroulera sous son propre poids.

\section{Henry Kistemaeckers}

Henry Kistemaeckers ${ }^{63}$, dont l'activité couvre le dernier quart du $\mathrm{XIX}^{\mathrm{e}}$ siècle, peut passer pour l'un des continuateurs de Lacroix \& Verboeckhoven. D'une inoxydable fidélité à ses convictions politiques et touché par la sévérité de la répression des Communards, il quitte son emploi de commissaire-comptable à la Compagnie Générale de Navigation de Londres pour ouvrir en 1875 une librairie à Bruxelles, pour y diffuser les livres de toute une série d'auteurs socialistes, républicains et démocrates, se faisant ainsi rapidement un nom parmi les librespenseurs et révoltés en tous genres. Les publications de la Librairie Contemporaine vont très vite éveiller la méfiance de la Justice à l'égard d'un éditeur qui présente lui-même sa maison en ces termes : « Maison de Commission pour

62 SACRÉ (J.), Les Mystères des bandes noires, cité dans FAYT (R.), Auguste Poulet-Malassis à Bruxelles (septembre 1863-mai 1871). Bruxelles, Les Libraires momentanément réunis, 1993 p. 110.

63 Colette BAUDET a consacré tout un ouvrage à cet éditeur important : Grandeurs et misères d'un éditeur belge : Henry Kistemaeckers (1851-1934). Bruxelles, Labor, coll. Archives du Futur, 1986. 
toute la librairie française, à l'exception toutefois des ouvrages religieux ou de piété, des traités, des brochures purement militaires, des écrits apologétiques des rois et des empereurs, des pontifes religieux ou civils et en général de tous ces corsaires de l'humanité » ${ }^{64}$.

Les difficultés qu'il va connaître sur le plan économique et dans ses relations avec les autorités auront raison de l'activisme politique de Kistemaeckers. Aussi, même s'il gardera des liens avec les milieux socialistes, dont il ne renoncera d'ailleurs jamais réellement à éditer des ouvrages, il se tourne dès 1880 vers la littérature. Le naturalisme lui offre la possibilité de poursuivre sur un autre terrain son combat contre les oppressions de toute sorte. Il lancera des débutants, souvent qualifiés de pornographes par la presse bien-pensante, et plus exposés que leurs aînés aux foudres de la Justice, qui évite de s'en prendre aux maîtres. Autour d'un clocher de Henry Fèvre et Louis Desprez, deux de ces débutants, sera ainsi à l'origine du plus grave procès intenté à l'éditeur dans une carrière pourtant fertile en démêlés judiciaires. Fèvre, mineur, échappe aux poursuites lancées par le Parquet de Paris ; Desprez et Kistemaeckers sont quant à eux condamnés à un mois de prison (peine épargnée à l'éditeur lorsqu'il comparaîtra en appel devant un nouveau jury) et à une lourde amende. Cité également par la Cour d'Assises du Brabant en décembre 1885, l'éditeur est acquitté par le Parquet de Bruxelles.

Durant cette même période, Kistemaeckers déploie ses activités en deux sens : d'une part celui des réimpressions galantes dans une présentation impeccable et avec des illustrations de Rops, réservées à un public de bibliophiles ; d'autre part en direction des auteurs groupés à 1'enseigne de La Jeune Belgique. Éthos bibliophilique d'un côté, détermination esthétique de l'autre, associée en sous-main à une certaine audace politique. Sous ce second égard, le mouvement amorcé par Lacroix \& Verboeckhoven en 1867 , de refonte et de relance réciproques d'une édition et d'une production littéraires nationales, semble se prolonger. Irait dans ce sens la publication en 1881 d'Un Mâle, de Camille Lemonnier. Mais s'il a, au départ, publié nombre d'auteurs associés au mouvement naturaliste ou y collaborant (Hannon et Nizet, notamment), Kistemaeckers ne sera cependant jamais leur éditeur en titre : leurs conceptions respectives de l'activité littéraire vont diverger. Eekhoud et Nizet seuls resteront fidèles à un homme pourtant combatif et entreprenant. La maison va donc péricliter : les jeunes naturalistes la quittent, les auteurs en vogue ne partagent plus ses idéaux esthético-politiques, et la crise qui touche le secteur de la librairie après 1885 se fait sentir dans tous les domaines, y compris celui des ouvrages pour bibliophiles. Les poursuites et condamnations à répétition dont Kistemaeckers est victime en Belgique viendront encore assombrir le tableau. Résultat : en 1903, il quitte définitivement la Belgique pour la France, « ruiné mais fier d'avoir préservé son indépendance $» 65$.

\section{Edmond Deman}

Il faut enfin faire sa place, dans ce rapide tour d'horizon de l'édition littéraire fin de siècle, à Edmond Deman. Non que cette place soit grande - en nombre d'ouvrages publiés, une cinquantaine, ni en durée d'activité, de 1888 à 1912 - , mais parce que l'éditeur des Poésies de Mallarmé (en 1899) a su en peu de temps lester son enseigne d'un solide crédit culturel. C'est surtout qu'avec cinquante ans de retard sur la France, la Belgique se dote avec lui d'une sorte de Curmer - d'un éditeur esthète, à la fois artiste et artisan, travaillant sur un pied d'égalité avec ses auteurs et ses illustrateurs, aussi attaché aux séductions de la typographie soignée et du papier de haut luxe qu'aux voluptés du texte raffiné. Deman, ce sera un catalogue peu fourni en titres mais dense en signatures prestigieuses, qu'elles soient de Belgique (Verhaeren, Gilkin, Maeterlinck, Crommelynck) ou de France (Villiers, Barbey d'Aurevilly, Bloy, Mallarmé, Ghil ou Gustave Kahn). Ce sera aussi, transversale à toutes ces signatures, une marque de prestige éditorial — une « griffe ». Ce sera, enfin, un micro-milieu culturel où l'élite du symbolisme communiera dans le sentiment partagé de sa propre excellence esthétique.

La brève trajectoire de Deman, libraire et éditeur, est aujourd'hui bien connue, grâce à 
l'étude approfondie qu'ont fait paraître Adrienne et Luc Fontainas ${ }^{66}$. On nous permettra donc de glisser sur l'anecdote pour aller à ce qui de notre point de vue constitue l'essentiel. Pour remarquer, d'abord, que l'éclat de sa maison, Deman ne le doit pas seulement à sa passion personnelle du beau livre et de la littérature d'exception, ni simplement au rôle actif de passeur joué par Émile Verhaeren, son principal auteur maison - pour lequel il réalise un tirage unique, sur soie, de son recueil Les Heures claires à l'occasion du Nouvel An 1897 -, mais aussi à une combinaison de facteurs institutionnels et économiques favorables. D'une part, Deman bénéficie de l'appel d'air créé par l'internationale symboliste telle qu'elle se met en place dans les années 1880 avec le mouvement de navette faisant voyager entre Paris et Bruxelles - physiquement ou par textes de cooptation réciproques - Bloy et Verhaeren, Mallarmé et Maeterlinck, René Ghil et Rodenbach. L'accueil fait aux uns à Paris est comme payé de retour par celui que Bruxelles, Liège ou Bruges font aux autres, et par les sollicitudes éditoriales que leur témoigne le patient, dévoué, désintéressé Edmond Deman. D'autre part, celui-ci tire parti - sans certes l'avoir cherché consciemment de l'état de saturation dans lequel est plongé autour de 1890 le système éditorial parisien, dont la machine tourne davantage au profit du roman psychologique ou des recueils de chroniques journalistiques que de la poésie éthérée pratiquée par les symbolistes, proies faciles des mercenaires de l'édition à compte d'auteur (Verlaine, Mallarmé en font les frais auprès de Lemerre puis de Vanier). Occasion historique d'inverser, au moins temporairement, l'hémorragie des auteurs d'un circuit éditorial à l'autre. Ainsi, comme l'a remarqué Paul Aron, « Mallarmé et Verlaine [et bien d'autres] trouvent [non seulement] en Belgique des tribunes, des critiques, un accueil dans le milieu mondain et artistique dont ils sont privés en France. [Mais] plus concrètement, les symbolistes français, et Mallarmé en particulier, trouvent en Belgique des conditions de publication exceptionnelles ${ }^{67}$. Pour ces auteurs en déshérence édito- riale, Deman représente une aubaine : celui qui confie que « l'édition, c'est ma danseuse » ${ }^{68}$ est peu regardant sur les coûts, les délais, et peu exigeant en matière de reconnaissance, chez ceux qui en bénéficient, des largesses qu'il leur fait. L'éditeur se ruine, mais engrange un capital de sympathie et de légitimité qui lui permettra d'apparaître, non pas comme un docile exécutant, mais comme un partenaire de "ses » auteurs. L'embellie sera brève — dès que l'édition parisienne se désengorge, au début du $\mathrm{xx}^{\mathrm{e}}$ siècle, les flux en direction de la Belgique se tarissent - , mais l'aura de Deman survivra à la disparition prématurée de sa maison.

Cas unique dans l'histoire des pratiques d'édition belges, du moins dans le secteur littéraire, Deman se confond, d'autre part, avec une griffe, supérieure à la série des ouvrages de qualité qu'il publie. Et cette griffe, il la fait figurer, sur son édition des Soirs de Verhaeren, non pas au bas de la page de couverture, mais au centre de cette page, comme pour afficher la conscience qu'il a d'être un véritable transfigurateur de l'œuvre - un « banquier symbolique » engageant sur chaque texte qu'il accueille le capital de confiance qu'il détient, auprès des auteurs et de ses pairs, dans le champ littéraire/éditorial. Pour intense qu'il soit, ce pouvoir de griffe ne doit cependant pas faire illusion : non seulement Deman publie peu, mais à très petit tirage, pour un public d'artistes et de bibliophiles, " gens, disait Mallarmé, qui ne lisent point ». Au tournant du siècle, sur le marché francobelge, ce sera bientôt l'heure de Grasset, de la NRF, du Mercure de France, de Fasquelle, autrement dit celle d'éditeurs conjuguant sans complexe exigence culturelle et préoccupations mercantiles, sens de l'œuvre et sens de la collection, goût du texte et pratique du livre esthétiquement quelconque. La beauté Deman n'a brillé qu'un laps, elle restera comme le souvenir d'une occasion magnifiquement manquée. C'est Casterman en Belgique, parmi quelques autres dans le même secteur, qui occupera le haut du pavé éditorial, géant aux pieds de plomb foulant la poussière d'or d'une multitude de petits éditeurs littéraires, hautement respectables

66 Edmond Deman éditeur (1857-1918). Art et édition au tournant du siècle. Bruxelles, Labor, coll. Archives du futur, 1997, 356 p. Voir sur cet ouvrage le compte rendu publié par l'un d'entre nous dans le présent numéro de Textyles.

67 Aron (P.), « Pour une description sociologique du symbolisme belge », dans Soncini FratTA (A.), éd., Le Mouvement symboliste en Belgique. Bologna, ClueB, 1990, p. 58. 
bien évidemment, mais récupérant la plupart du temps les laissés-pour-compte du système éditorial français ou ceux qui en sont revenus, les poètes en surnombre, les écrivains régionalistes, quand ils ne donnent pas leur première chance à tels qui, publiant à Bruxelles, lorgnent déjà l'horaire des trains pour Paris.

\section{Conclusions}

Au moment où l'édition belge s'apprête à entrer dans le $\mathrm{xx}^{\mathrm{e}}$ siècle, son identité est pour le moins fragile. En Belgique, à la fin du XIX $\mathrm{X}^{\mathrm{e}}$ siècle, on produit avant tout de la belle ouvrage - au service de l'œuvre certes, ainsi chez Deman -, mais en plaquant celle-ci sur un support dont le pouvoir de diffusion sera inversement proportionnel au luxe apporté à sa fabrication matérielle. Ce qui se transmet de génération en génération depuis Plantin, c'est non pas une représentation littéraire du métier, mais une représentation graphique, au principe de ce que nous appelons un " habitus techniciste », convertible le cas échéant du côté de la bibliophilie. Cette disposition imprègne les esprits, fonde les choix, soustend les jugements. Les livres, les hommes et les maisons sont appréciés à partir de catégories dérivées du métier d'imprimeur, qualité du papier et de l'illustration, élégance typographique, etc. L'éditeur moderne reste encore à inventer. Est-ce un hasard ou un bégaiement de l'histoire si le seul prix important que se décernent annuellement les éditeurs belges, aujourd'hui encore, s'appelle le « Prix PlantinMoretus » (et non, par exemple, le Prix Deman)?

Ces imprimeurs, de Plantin à Casterman, sont passés maîtres dans l'art d'équilibrer coûts et coups. C'est une sage philosophie, que 1'on recommanderait aujourd'hui encore à tout débutant. Mais ce n'est certainement pas une attitude favorisant l'éclosion de nouveaux talents ou de nouveaux genres. On remarque d'ailleurs que si ces maisons se perpétuent de père en fils ou d'oncle en neveu, c'est en diminuant toujours le nombre de coups pour toujours mieux contrôler les coûts. Au point que ces maisons laissent peu à peu s'atrophier leur fonction éditoriale, lorsqu'elle existe, au profit de leur fonction graphique. Et ce qui se lègue d'une génération à l'autre, c'est moins un capital symbolique qu'une entreprise, avec ses machines et ses stocks de papier. On remarquera, d'autre part, que les imprimeurs ou les éditeurs belges qui maîtrisent mal le rapport entre coups et coûts n'ont pas en général l'occasion de transmettre leur maison à leurs descendants. Icare après la chute, Job incriminant l'ingratitude de Dieu, ils sont sur la paille dans leur vieillesse, écœurés, dégoûtés, remplis en tout cas de ressentiment, avec le sentiment d'avoir trop payé, en vain, de leur personne, de leur temps et de leur bourse. Bassompierre comme Deman, Plomteux comme Kistemaeckers manquent de ce « sens du jeu » qui fait que Casterman est encore présent de nos jours sur la place. Ils ont sans doute produit plus d'ouvrages originaux et importants dans l'histoire culturelle belge que Casterman (encore que Tintin, dans son genre, vaille bien l'Encyclopédie ou Verhaeren dans le leur), mais ils n'ont pas tenu plus d'une génération. C'est là sans doute une des caractéristiques de l'édition belge depuis ses « origines » : celle qui vit sur le plan culturel est célibataire; celle qui survit sur le plan économique est familiale.

La fragilité de l'édition belge à l'aube du $\mathrm{xx}^{\mathrm{e}}$ siècle tient aussi au fait qu'elle n'est pas encore très belge. Comment le serait-elle d'ailleurs ? Non seulement le pays lui-même est encore jeune, mais ses éditeurs se sont longtemps inscrits dans des pratiques « extra-territoriales ». La plus patente est évidemment la contrefaçon, qui remonte à une époque où n'existaient encore que des villes, principautés et empires laissant à chacun la liberté de tirer profit des opportunités offertes par la porosité des frontières avec la France. La Belgique instaurée depuis trente ans, les imprimeurs-« éditeurs » continuent pourtant à se comporter comme si elle n'existait pas. Pourquoi l'auraient-ils fait - pourrait-on rétorquer alors que la notion de propriété intellectuelle n'était pas encore entrée dans la loi belge ? Parce qu'ils auraient pu avoir, à l'instar d'acteurs culturels dans d'autres pays jeunes (la Grèce, l'Italie), une conscience nationale et la volonté collective de contribuer à l'émergence, par exemple, d'une littérature spécifiquement belge. Cette proposition fait sourire : comme si un imprimeur-éditeur eût pu concevoir pareil dessein intellectuel ambitieux, généreux ou « idéaliste » en Belgique au milieu du siècle passé... De fait, les imprimeurs-éditeurs belges du $\mathrm{XIX}^{\mathrm{e}}$ siècle n'appartiennent pas au champ culturel, scientifique ou politique de la nation naissante; ils relèvent de l'artisanat ou de l'industrie, sans 
autre objectif que la rentabilité économique rapide. Ils s'opposent en cela aux quelques éditeurs littéraires dont la Belgique se dotera, pauvrement, dans le dernier tiers du siècle (Lacroix \& Verboeckhoven, Kistemaeckers, Deman), mais dont les ambitions culturelles, quoi qu'il y paraisse et quelque provocante que soit cette observation, relèveront davantage d'une logique française (publier Hugo, Lautréamont, Mallarmé, Villiers, Ghil pour afficher une légitimité d'emprunt) que d'une logique spécifiquement belge.

Les relations privilégiées que nos plus importants imprimeurs-éditeurs ont entretenues avec l'Église catholique ont sans doute également contribué à les tenir à distance d'un investissement dans la construction d'une identité littéraire/éditoriale forte. Les livres religieux partaient dans le monde entier, se vendaient comme des petits pains miraculeusement multipliables. Que pouvait dès lors représenter le marché local pour Casterman ou Dessain ? À peine quelques milliers de livres scolaires. Et dès les années trente, les albums de Tintin prendront le relais des missels pour diffuser planétairement sainte parole et saine morale. Le sol de la Belgique ne sera qu'un pied-à-terre pour les grands imprimeurs-éditeurs qui s'y sont développés à la faveur des hasards de l'histoire et du capitalisme industriel ambiant ; ce ne sera qu'un horizon restreint pour les petits imprimeurs-éditeurs locaux dont les produits sortiront rarement du territoire. S'opère ainsi un clivage entre une poignée de maisons au rayonnement si international qu'il passe au-dessus de la Belgique et une multitude de petites maisons provinciales au rayonnement si faible qu'il couvre à peine le pays. Et les quelques rares éditeurs littéraires bruxellois qui échappent à cette polarisation relèvent, à distance respectueuse, du champ culturel français.

C'est dire combien la persistance de la contrefaçon jusqu'en 1854 a agi en profondeur sur l'édition belge. Injectée dans le trinôme Imprimerie-Famille-Église qui gouverne l'édition sur nos territoires depuis Plantin, elle installe des rapports problématiques entre la France et la Belgique, dont les effets se font encore sentir un siècle plus tard. Insistons-y encore : la contrefaçon a construit une disposition au sein de l'édition belge qui la porte à reproduire ce que la France produit. Il ne s'agit pas seulement de reproduction graphique : toutes les fonctions éditoriales créatrices vont se trouver comme inhibées pour longtemps en Belgique parce que c'est Paris, toujours et encore, qui imposera ses choix, ses valeurs, ses modes. Les créateurs descendront dès lors, en y «montant ", sur la capitale française, et l'édition belge n'aura plus qu'à se tourner vers les domaines qui n'en demandent pas - des catéchismes aux livres de cuisine, des manuels scolaires aux traités de jardinage ${ }^{69}$. La contrefaçon, certes, a renforcé chez nos imprimeurs-éditeurs le sens du coup très rapide ainsi que leur grande compétence technique. Mais ce double bénéfice se paiera d'une durable incapacité à travailler en rythme de phase avec la France - par exemple à œuvrer en même temps qu'elle au développement de l'éditeur comme cocréateur du texte-livre, à la manière de Curmer.

Tout semble en définitive s'être passé comme si la Belgique, d'être restée trop longtemps sur les deux quais de la contrefaçon puis de l'édition religieuse, avait raté le train de l'Histoire. L'éditeur émerge en France, vers 1840, en synchronie avec la figure romantique de l'auteur et en phase avec une littérature vivante,

69 L'hésitation du jeune État belge à supprimer la contrefaçon montre aussi combien la chose culturelle est étrangère à l'autorité publique au XIX ${ }^{e}$ siècle. En fait, l'édition ne relève pas de la « culture " (terme jamais employé dans cette acception à l'époque), mais de la sphère économique, où l'État n'interviendra pas encore. Cette attitude de neutralité vis-à-vis de l'édition du livre, c'est-à-dire son classement dans les matières économiques sans intérêt national, persistera jusqu'au milieu du $\mathrm{xx}^{\mathrm{e}}$ siècle. Et il faudra attendre la fin des années soixante et l'émergence d'un Ministère de la Culture autonome vis-à-vis du Ministère de l'Éducation nationale, pour que l'État belge envisage timidement de s'intéresser à l'édition comme activité culturelle autant qu'économique. Aujourd'hui encore, c'est l'édition littéraire qui intéresse au premier chef les services spécialisés de la Communauté française. La France, entre-temps, a développé des structures d'encadrement très solides, à la fois au sein de la profession elle-même (Syndicat national de l'Édition, Cercle de la Librairie, etc.), et au sein de l'État (Direction du Livre, Caisse nationale des Lettres, etc.). Celui-ci a intégré depuis longtemps la conception du livre comme bien économique particulier (e.g. prix unique). En Belgique, le livre restera essentiellement livré à lui-même, comme au bon vieux temps de la contrefaçon. 
conquérante, de rayonnement international. Rien de tout cela au Nord: pas de littérature vivace avant De Coster et Lemonnier, pas non plus d'éditeurs créatifs. Et le relais de l'Église, qui sauve de la faillite le secteur de l'imprimerie, l'enfonce encore pour longtemps dans de semblables pratiques de reproduction. Lorsque la Belgique enfantera enfin ses Curmer, sous l'espèce des Deman et Kistemaeckers, ce sera avec un retard d'une génération. Au même mo- ment, la France - où les éditeurs-artistes appartiennent déjà au passé quand ils ne se maintiennent pas en province - sera déjà en train de passer à l'étape suivante, en engendrant de puissantes entreprises culturelles, fondée sur une séparation radicale entre édition et imprimerie, et une représentation du livre comme objet secondaire et graphiquement quelconque, mais mis au service d'un texte esthétiquement ou intellectuellement premier ${ }^{70}$.

70 Lorsque, au milieu du $\mathrm{xx}^{\mathrm{e}}$ siècle, l'édition belge - ou plutôt l'imprimerie éditoriale belge - va précéder la France en matière de production du livre de poche, avec la reprise par André Gérard de la formule du pocket book à l'américaine, elle va se révéler incapable à la fois de l'assumer au-delà d'une première phase d'expansion, puisque le passage aux grosses cadences industrielles coulera Marabout au début des années septante, et de faire respecter sa place dans les histoires de l'édition de langue française (où l'initiative Hachette l'emportera sur l'initiative Marabout, pourtant en avance de quatre ans sur elle). 